\title{
GROUPE FONDAMENTAL DE L'ESPACE DES FEUILLES DANS LES FEUILLETAGES SANS HOLONOMIE
}

\author{
GILBERT LEVITT
}

\begin{abstract}
Résumé
On étudie les feuilletages $\mathscr{F}$ de codimension 1 , de classe $C^{2}$, sans holonomie(notamment ceux définis par une 1-forme différentielle fermée), dans les deux contextes suivants: feuilletages non singuliers des variétés ouvertes, feuilletages à singularités de Morse des variétés fermées. Le principal outil est un quotient de $\pi_{1} M$, le "groupe fondamental de l'espace des feuilles" $\pi_{1}(M / \mathscr{F})$. Tout sous-groupe de type fini de $\pi_{1}(M / \mathscr{F})$ est un produit libre de groupes abéliens libres, et les facteurs de rang $\geq 2$ s'interprètent géométriquement. On en déduit en particulier l'absence de feuille exceptionnelle, sous certaines hypothèses sur $\pi_{1} M$ (par exemple: $\pi_{1} M$ est de type fini et n'a pas de quotient libre non abélien). Ces résultats sont appliqués aux feuilletages (non singuliers) transversalement affines, et aux actions libres sur les variétés (non séparées) de dimension 1 . On détermine également la structure transverse d'un feuilletage défini par une 1 -forme fermée singulière générique sur $M$ fermée.
\end{abstract}

\section{Introduction}

Soit $\mathscr{F}$ un feuilletage transversalement orientable de codimension 1 sur une variété fermée $M$. Si $\mathscr{F}$ est de classe $C^{2}$ et sans holonomie, un célèbre théorème de Sacksteder [19] affirme que $\mathscr{F}$ est topologiquement conjugué à un feuilletage défini par une 1-forme différentielle fermée; en particulier les feuilles sont toutes compactes ou toutes denses, et $M$ fibre sur le cercle [25].

On est donc conduit à considérer les feuilletages sans holonomie dans des cadres plus généraux: feuilletages singuliers sur les variétés fermées, ou feuilletages sur les variétés ouvertes. Pour étudier par exemple les feuilletages transversalement affines sur $M$ compacte, nous utiliserons les feuilletages sans holonomie induits sur des revêtements convenables (non compacts) de $M$.

La structure d'un feuilletage sans holonomie peut alors être très compliquée (même si $\mathscr{F}$ est $C^{\infty}$, ou analytique, ou défini par une 1-forme

Received June 9, 1988. 
fermée). En particulier [3], [10], il peut exister des feuilles exceptionnelles (ni propres ni localement denses).

Notre outil principal pour étudier un tel feuilletage sera ce que nous appellerons le groupe fondamental de l'espace des feuilles $\pi_{1}(M / \mathscr{F})$, que l'on peut définir comme le $\pi_{1}$ de l'espace classifiant $B \Gamma$ introduit par Haefliger (cf. [7]) pour le pseudogroupe d'holonomie $\Gamma$.

D'une façon générale, il nous semble intéressant de rechercher les liens entre les propriétés d'un feuilletage $\mathscr{F}$ et des invariants de topologie algébrique du classifiant $B \Gamma$, en particulier son $\pi_{1}$.

Le $\pi_{1}$ de $B \Gamma$ est en effet l'invariant le plus accessible, car il est susceptible d'une définition directe (cf. [20]): $\pi_{1}(M / \mathscr{F})$ est le quotient de $\pi_{1} M$ par le sous-groupe distingué $\mathscr{L}$ engendré par les classes d'homotopie libre des lacets contenus dans des feuilles et à holonomie triviale (cf. cidessous $§ 0 . \mathrm{A})$. D’ailleurs, si $\mathscr{F}$ est sans holonomie, le classifiant $B \Gamma$ est un $K(\pi, 1)$ d'après un résultat de Haefliger.

Pour un feuilletage sans holonomie, le groupe $\pi_{1}(M / \mathscr{F})$ est lié à la fois à $\pi_{1} M$ (dont il est un quotient), aux propriétés géométriques du feuilletage (feuilles localement denses, exceptionnelles, etc.), et, dans le cas d'une 1-forme fermée singulière sur une variété compacte, à certains entiers associés au feuilletage. Donnons quelques exemples.

Soit $\mathscr{F}$ transversalement orientable, de classe $C^{2}$, sans holonomie, disons sur une variété $M$ dont le $\pi_{1}$ est de type fini. Un de nos principaux résultats sera que $\pi_{1}(M / \mathscr{F})$ est toujours un produit libre de groupes abéliens libres. Nous verrons d'autre part qu'un sous-groupe abélien mais non cyclique de $\pi_{1}(M / \mathscr{F})$ détecte la présence de feuilles localement denses d'un type bien particulier.

Lorsque $\pi_{1} M$ n'a pas de quotient libre non abélien, ceci nous permettra de comprendre la structure transverse du feuilletage. Nous obtiendrons par exemple un théorème "à la Sacksteder" (absence de feuilles exceptionnelles) pour les feuilletages à singularités de Morse des variétés fermées.

Mentionnons deux autres applications de ce type d'arguments. Soit $\mathscr{F}$ un feuilletage (non singulier) transversalement affine sur une variété fermée $M$. Il existe un revêtement abélien libre naturel de $M$ sur lequel le feuilletage induit est défini par une 1-forme fermée, donc sans holonomie.

On en déduit, lorsque $\pi_{1} M$ ne contient pas de sous-groupe libre non abélien, un théorème de structure pour $\mathscr{F}$ qui montre en particulier l'absence de feuilles exceptionnelles; s'il existe une courbe transverse coupant toute feuille, les feuilles sont en fait denses (à moins que $\mathscr{F}$ ne soit donné par une fibration sur $S^{1}$ ). 
Soit d'autre part $G \subset \operatorname{Diff}_{+}^{2}(V)$ un groupe de type fini agissant librement sur une variété $V$ de dimension 1 (non séparée) connexe et simplement connexe. Ce groupe est isomorphe à $\pi_{1}(M / \mathscr{F})$, où $\mathscr{F}$ est un feuilletage sans holonomie sur une variété ouverte $M$, et est donc un produit libre de groupes abéliens libres. Nous espérons arriver à généraliser ce genre d'arguments pour pouvoir comprendre les groupes agissant librement sur les arbres réels ( $\mathbb{R}$-arbres au sens de [16]).

Pour un feuilletage défini par une 1-forme fermée $\omega$ à singularités de Morse sur une variété fermée, l'étude de $\pi_{1}(M / \omega)$ permettra de déterminer la structure transverse dans le cas générique. Elle conduira d'autre part à définir à partir de $\pi_{1}(M / \omega)$ deux nombres $d$ et $b$, que nous relierons respectivement au nombre de singularités d'indice 1 de $\omega$ et au nombre minimal de générateurs du pseudogroupe d'holonomie. Comme application, nous verrons par exemple que, sur la somme connexe de $p+1$ exemplaires de $S^{1} \times S^{n-1} \quad(n \geq 3)$, toute forme fermée de Morse a au moins $p$ singularités d'indice 1 (resp. $n-1$ ).

Ce travail a été commencé lors d'un séjour à l'université de Genève, que je remercie pour son hospitalité.

\section{Table des matieres}

Plan de l'article et énoncé des résultats

0 . Préliminaires

$0 . A$. Groupe fondamental de l'espace des feuilles

0. B. Définitions et notations

0.C. Proligals

I. Formes de Morse sur les variétés fermées

I.A. Détermination de $\pi_{1}(M / \omega)$

I.B. Formes $\pi_{1}$-stables

I.C. Invariant $d$ et singularités coniques

I.C.i Calcul de $d(\omega)$

I.C.ii Persistance de singularités coniques

I.C.iii Quelques inégalités sur $d(\omega)$

I.D. Générateurs du pseudogroupe d'holonomie

II. Variétés ouvertes

II.A. Sous-groupes de type fini de $\pi_{1}(M / \omega)$

II.B. Composantes faiblement complètes et sous-groupes abéliens

II.C. Formes faiblement complètes

III. Application aux feuilletages affines

III.A. Variétés compactes

III.B. Variétés non compactes

IV. Généralisation aux feuilletages sans holonomie

IV.A. Quelques conséquences de Sacksteder

IV.B. Relations avec le $\pi_{1}$

V. Application aux variétés non séparées de dimension 1

Références 
Les sections I.B à I.D ne sont pas utilisées ultérieurement.

\section{Plan de l'article et énoncé des résultats}

0. Nous commençons par une discussion informelle, sans démonstrations, du $\pi_{1}$ de l'espace des feuilles dans le cas particulier d'un feuilletage défini par une 1-forme fermée non singulière $\omega$. Cette section est inspirée par des idées de Haefliger, et ne contient rien de réellement nouveau. Elle n'est pas utilisée stricto sensu dans la suite de l'article, mais justifie et met en perspective la définition $\pi_{1}(M / \mathscr{F})=\pi_{1} M / \mathscr{L}$.

Après quelques définitions, nous démontrons un résultat algébrique sur les produits libres de groupes abéliens libres (Lemme 0), qui nous sera utile par la suite.

I. Dans la première partie de l'article proprement dit, nous considérons une 1-forme fermée $\omega$ à singularités de Morse sur une variété fermée orientable $M^{n}$ de dimension $n \geq 3$. Pour simplifier, considérons d'abord des formes minimales, c'est-à-dire définissant un feuilletage dont toute feuille est dense.

On peut considérer deux types d'exemples. D'abord des formes non singulières, ou plus généralement sans singularités d'indice 1 ou $n-1$. Pour de telles formes, les intersections des feuilles avec une courbe fermée transverse quelconque $C$ sont les orbites de l'action sur $C$ d'un groupe de rotations.

Les formes possédant cette propriété ont été appelées faiblement complètes dans [13]; nous y avons montré en particulier que, si $\pi_{1} M$ n'a pas de quotient libre non abélien, toute forme minimale $\omega$ est faiblement complète, et, sans hypothèse sur $\pi_{1} M$, que toute forme non exacte est cohomologue à une forme minimale faiblement complète.

On construit d'autres exemples à partir des échanges d'intervalles minimaux du cercle (cf. $[1, \S 1.4]$ ). Les intersections des feuilles avec une courbe transverse sont alors d'un type très différent: si $C$ est convenablement choisie, il n'existe pas de difféomorphisme globalement défini $\varphi: C \rightarrow C$ tel que $x$ et $\varphi(x)$ soient sur la même feuille $\forall x$ (à part $\varphi=$ id).

Géométriquement, ceci est dû à l'existence de singularités d'indice 1 ou $n-1$ dont les deux bouts singuliers (cf. [13] ou 0.B.iii; voir Figure 2) ne sont pas dans la même feuille; nous qualifions ces singularités de bloquantes, car elles font obstacle à l'extension des applications d'holonomie définies localement sur $C$.

Selon qu'elles sont ou non faiblement complètes, les formes se comportent différemment lorsqu'on les approche par des formes qui leur sont 
cohomologues [13]: si $\omega$ est minimale et faiblement complète, il en est de même de ses perturbations; au contraire, une forme minimale non faiblement complète peut être approchée dans sa classe de cohomologie par des formes dont toute feuille régulière est compacte.

Cette différence se répercute sur le $\pi_{1}$ de l'espace des feuilles: si $\omega$ est faiblement complète, alors $\pi_{1}(M / \omega)$ est isomorphe au groupe des périodes $P(\omega) \subset \mathbb{R}$, donc abélien libre; par contre, si $\omega$ est minimale mais pas faiblement complète, nous montrerons que $\pi_{1}(M / \omega)$ est libre non abélien, comme pour les formes cohomologues à feuilles compactes qui approchent $\omega$.

Considérons maintenant une forme de Morse quelconque $\omega$, pas forcément minimale. On sait ([9], appendice de [1]) que ses feuilles non fermées (dans $M-\operatorname{Sing} \omega$ ) se répartissent en un nombre fini d'ouverts saturés $U_{i}$, dits composantes minimales car toute feuille contenue dans un $U_{i}$ y est dense; en particulier, il n'y a pas de feuille exceptionnelle. En considérant l'intersection des feuilles avec une courbe transverse $C_{i} \subset U_{i}$, on peut parler de composante minimale faiblement complète. On a alors:

Théorème 1. Le groupe $\pi_{1}(M / \omega)$ est un produit libre de groupes abéliens libres. Les facteurs de rang $\geq 2$ correspondent bijectivement aux composantes minimales faiblement complètes de $\omega$.

Le résultat est assez naturel si toutes les composantes minimales sont faiblement complètes. Pour le montrer dans le cas général, nous perturberons $\omega$ dans sa classe de cohomologie de façon à remplacer les composantes non faiblement complètes par des feuilles compactes, et grâce au Lemme 0 nous montrerons que cette perturbation ne change pas $\pi_{1}(M / \omega)$.

Nous dirons qu'une forme $\omega$ est $\pi_{1}$-stable si $\pi_{1}\left(M / \omega^{\prime}\right) \simeq \pi_{1}(M / \omega)$ pour $\omega^{\prime}$ cohomologue à $\omega$ et assez proche de $\omega$. Nous verrons que génériquement une forme est $\pi_{1}$-stable, et que les composantes minimales d'une forme $\pi_{1}$-stable sont faiblement complètes. Nous en déduirons:

Théorème 2. Munissons l'espace des 1-formes fermées de Morse sur $M$ de la topologie $C^{r}(1 \leq r \leq \infty)$. Génériquement dans cet espace, toutes les composantes minimales de $\omega$ sont faiblement complètes.

Dans un prochain article, nous étudierons les formes qui ne sont pas $\pi_{1}$ stables, et nous verrons qu'elles appartiennent à des hypersurfaces linéaires par morceaux.

La détermination de $\pi_{1}(M / \omega)$ nous permet d'attacher deux entiers à $\omega$ : nous noterons $d(\omega)$ le nombre de facteurs du produit libre $\pi_{1}(M / \omega)$, diminué de 1; d'autre part, $b(\omega)$ sera le rang de l'abélianisé de $\pi_{1}(M / \omega)$, ou encore le nombre minimal de générateurs de $\pi_{1}(M / \omega)$.

L'invariant $d$ est relié au nombre de singularités de $\omega$. Pour simplifier, 
nous n'énonçons ici le résultat que dans un cas particulier.

Théorème 3. Soit $\omega$ de Morse, sans singularité d'indice 0 (resp. n) et sans liaison entre singularités d'indice 1 (resp. $n-1)$. Alors $d(\omega)$ est égal au nombre de singularités bloquantes d'indice 1 (resp. $n-1)$.

Ce théorème peut servir à majorer le nombre de singularités bloquantes de $\omega$ : si $\pi_{1} M$ n'a pas de quotient libre de rang $k$, une forme $\omega$ comme dans le Théorème $3 \mathrm{a}$ au plus $k-2$ singularités bloquantes d'indice 1 (resp. $n-1)$. En sens contraire, toute forme non exacte est cohomologue à une forme sans singularité bloquante [13, Théorème III.1]; on a cependant:

Théorème 4. Si $\omega^{\prime}$ est cohomologue à $\omega$, elle a au moins $d(\omega)$ singularités d'indice 1 (resp. $n-1$ ).

Ce théorème s'applique par exemple sur les variétés dont le $\pi_{1}$ est libre: sur une telle variété, le nombre de singularités d'indice 1 (resp. $n-1$ ) d'une forme fermée de Morse est au moins égal au premier nombre de Betti de $M$.

L'invariant $b(\omega)$ est quant à lui relié au nombre minimal de générateurs du pseudogroupe d'holonomie de $\omega$. Considérons par exemple une forme $\omega$ minimale non faiblement complète, et soit $C$ une courbe transverse. La relation d'équivalence donnée sur $C$ par "être sur la même feuille" n'est pas définie par l'action d'un groupe; elle peut être engendrée par l'identification, par des rotations, d'intervalles ouverts de $C$ (correspondant au domaine maximal de définition des applications d'holonomie). On peut montrer qu'il faut au moins $b(\omega)-1$ paires d'intervalles pour engendrer le pseudogroupe d'holonomie de $\omega$ sur $C$. Réciproquement:

Théorème 5. Supposons qu'il existe une courbe fermée transverse rencontrant toute feuille de $\omega$. Alors il en existe aussi une sur laquelle le pseudogroupe d'holonomie est engendré par $b(\omega)-1$ identification de paires d'intervalles ouverts.

II. Dans la deuxième partie, nous considérons une 1-forme fermée non singulière $\omega$ sur une variété $M^{n}$ ouvert orientable sans bord, de dimension quelconque. Notre premier résultat est le suivant:

Théorème 6. Tout sous-groupe de type fini $H \subset \pi_{1}(M / \omega)$ est un produit libre de groupes abéliens libres.

La démonstration consiste à se ramener au Théorème 1 en identifiant $H$ à $\pi_{1}\left(V / \omega_{V}\right)$, où $V^{n-1}$ est une variété fermée et $\omega_{V}=i^{*} \omega$ est une forme fermée de Morse induite par une immersion $i: V \rightarrow M$.

Nous généralisons ensuite la deuxième assertion du Théorème 1 , la notion de composante faiblement complète s'étendant facilement à une forme sur une variété non compacte (voir 0.B.ii).

Soit $U$ une composante faiblement complète de $\omega$. On montre que 
l'image $P_{U}$ de $\pi_{1} U$ dans $\pi_{1}(M / \omega)$, bien définie à conjugaison près, est un sous-groupe abélien; en fait, l'homomorphisme $\rho: \pi_{1}(M / \omega) \rightarrow \mathbb{R}$, induit par l'intégration de $\omega$ sur les lacets, définit un isomorphisme de $P_{U}$ avec le sous-groupe dense $P(\omega \mid U) \subset \mathbb{R}$.

Théorème 7. L'application ci-dessus définit une bijection entre les composantes faiblement complètes de $\omega$, et les classes de conjugaison de sousgroupes abéliens maximaux $P \subset \pi_{1}(M / \omega)$ avec $\rho(P)$ dense dans $\mathbb{R}$.

Nous caractérisons ensuite les formes pour lesquelles $\pi_{1}(M / \omega)$ est isomorphe à $P(\omega)$, que nous appelons formes faiblement complètes. Pour simplifier, nous nous limitons ici au cas où $P(\omega)$ est dense dans $\mathbb{R}$ (c'està-dire de rang $\geq 2$ ) .

Théorème 8. Soit $\omega$ une forme avec $P(\omega)$ dense dans $\mathbb{R}$. Les conditions suivantes sont équivalentes:

$-\pi_{1}(M / \omega)$ est isomorphe à $P(\omega)$;

$-\pi_{1}(M / \omega)$ ne contient pas de groupe libre non abélien;

- $\omega$ possède une et une seule composante faiblement complète $U$; toute feuille $L \subset M-U$ est fermée, sépare $M$ en deux composantes, et $\omega$ est exacte dans la composante de $M-L$ qui ne contient pas $U$.

Si $\omega$ vérifie ces conditions, toute feuille est fermée ou localement dense. Nous en déduirons:

Corollaire. Si $\pi_{1} M$ ne contient pas de groupe libre non abélien, ou si $\pi_{1} M$ est de type fini et n'a pas de quotient libre non abélien, alors une forme fermée sur $M$ ne peut pas avoir de feuille exceptionnelle.

On sait [10] qu'une forme fermée sur une variété ouverte $M$ peut avoir des feuilles exceptionnelles. On ignore toutefois si ce phénomène peut se produire lorsque $\pi_{1} M$ est de type fini.

III. Soit $\mathscr{F}$ un feuilletage (non singulier) transversalement affine de codimension 1 sur une variété fermée $M$. La structure affine définit un homomorphisme d'holonomie $\pi$ de $\pi_{1} M$ dans le groupe affine $\mathrm{Aff}^{+}(\mathbb{R})=$ $\{x \mapsto a x+b ; a>0\}$ (nous supposons $\mathscr{F}$ transversalement orientable).

Lorsque le groupe d'holonomie $H=\operatorname{image}(\pi)$ ne se compose que de translations, le feuilletage peut être défini par une 1-forme fermée. Plus généralement, on comprend bien les feuilletages dont l'holonomie $H$ est abélienne [21]; on sait en particulier qu'ils n'ont pas de feuille exceptionnelle, ce qui n'est pas toujours vrai dans le cas général (Hector).

Supposons donc que $H$ n'est pas abélien, et considérons le revêtement abélien libre $N \rightarrow M$ correspondant à l'homomorphisme d'holonomie linéaire (un lacet $\alpha$ se relève à $N$ si et seulement si $\pi(\alpha)$ est une translation). Le feuilletage induit sur $N$ est défini par une forme fermée $\omega$ avec $P(\omega)$ dense. Si $\pi_{1} M$ n'a pas de sous-groupe libre non abélien, il en est de 
même de $\pi_{1}(N / \omega)$, et on peut appliquer le Théorème 8 . On en déduit:

Théorème 9. Soit $\mathscr{F}$ un feuilletage transversalement orientable, transversalement affine, sur une variété fermée $M$ dont le $\pi_{1}$ ne contient pas de groupe libre non abélien. On suppose que l'holonomie $H$ de $\mathscr{F}$ n'est pas abélienne. Alors:

-Il existe un ouvert saturé non vide $V$ tel que toute feuille contenue dans $V$ y est dense. L'application (ensembliste) naturelle $V / \mathscr{F} \rightarrow \mathbb{R} / H$ est une bijection $(\mathbb{R} / H$ est l'ensemble des orbites pour l'action affine de $H$ sur $\mathbb{R})$.

-La frontière de $V$ se compose d'un nombre fini de feuilles compactes $L_{i}$. Chaque $L_{i}$ sépare $M$. Dans la composante de $M-L_{i}$ qui ne contient pas $V$, le feuilletage est à holonomie abélienne (composée uniquement d'homothéties ).

Corollaire. $\mathscr{F}$ n'a pas de feuille exceptionnelle.

Corollaire. S'il existe une courbe fermée transverse coupant toute feuille, alors toute feuille est dense et $M / \mathscr{F}$ est en bijection avec $\mathbb{R} / H$.

Il peut exister des feuilles localement denses autres que celles de $V$, mais elles ne sont pas de même nature: les feuilles de $V$ sont à croissance exponentielle, alors que dans $M-V$ toute feuille est à croissance polynomiale.

Le Théorème 9 reste vrai si $M$ n'est pas compacte, à ceci près que la frontière de $V$ peut se composer d'une famille discrète (donc dénombrable, mais pas forcément finie) de feuilles fermées. Joint au corollaire du Théorème 8 , ceci nous permettra de montrer:

Théorème 10. Soit $\mathscr{F}$ un feuilletage transversalement affine sur une variété $M$, compacte ou non. Si $\pi_{1} M$ ne contient pas de sous-groupe libre non abélien, alors $\mathscr{F}$ n'a pas de feuille exceptionnelle.

$\mathrm{T}$. Traba et $\mathrm{S}$. Matsumoto ont remarqué que ce théorème se généralise aux feuilletages transversalement projectifs.

IV. Dans la partie IV, nous étendons les résultats des parties I et II aux feuilletages de classe $C^{2}$, sans holonomie. D'après le théorème de Sacksteder [19], un feuilletage non singulier d'une variété fermée n'a pas de feuille exceptionnelle. Par contre, il peut exister des feuilles exceptionnelles dans un feuilletage à singularités de Morse sur une variété fermée (on construit des exemples sur $\left(S^{1} \times S^{n-1}\right) \#\left(S^{1} \times S^{n-1}\right)$ à partir de [3]), et dans un feuilletage non singulier sur une variété ouverte à $\pi_{1}$ abélien [10].

Grâce à la partie II et à [19], nous montrerons, comme cas particulier d'un résultat sur les variétés ouvertes (Théorème 15 ci-dessous):

Théorème 11. Soit $\mathscr{F}$ un feuilletage transversalement orientable, sans 
holonomie, de classe $C^{2}$, à singularités de Morse, sur une variété fermée orientable $M^{n}(n \geq 3)$. Si $\pi_{1} M \quad n^{\prime}$ 'a pas de quotient libre non abélien, alors $\mathscr{F}$ n'a pas de feuille exceptionnelle.

On peut de plus dire exactement quand $\mathscr{F}$ est topologiquement conjugué à un feuilletage défini par une 1-forme fermée.

Sans hypothèse sur $\pi_{1} M$, on a:

Théorème 12. Soit $\mathscr{F}$ comme dans le Théorème 11. On peut, en modifiant $\mathscr{F}$ au voisinage de ses singularités, l'approcher par un feuilletage topologiquement conjugué à une 1-forme fermée (donc sans feuille exceptionnelle).

Il nous semble vraisemblable que la propriété d'être conjugué à une 1-forme fermée soit générique.

Du Théorème 12 on déduit:

Proposition. Soit $\mathscr{F}$ comme dans le Théorème 11. Supposons que $\mathscr{F}$ est de la forme $i^{-1}(\mathscr{G})$, où $i: M^{n} \rightarrow N^{n+1}$ est une immersion de $M$ dans une variété ouverte munie d'un feuilletage (non singulier) $\mathscr{G}$ de classe $C^{2}$ sans holonomie. Alors $\mathscr{F}$ est topologiquement conjugué à une 1-forme fermée.

Cette proposition nous permettra d'étudier les feuilletages non singuliers de classe $C^{2}$, sans holonomie, transversalement orientables, sur une variété $N$ (compacte ou non). Nous montrerons d'abord le fait suivant, conjecturé par Lamoureux [12].

Théorème 13. Un feuilletage $\mathscr{G}$ comme ci-dessus n'admet pas de transversale fermée homologue à 0 .

Nous généraliserons également le Théorème 6.

Théorème 14. Tout sous-groupe de type fini $H \subset \pi_{1}(N / \mathscr{G})$ est un produit libre de groupes abéliens libres.

On pourrait d'ailleurs analyser plus précisément la structure de $\pi_{1}(N / \mathscr{G})$, et généraliser les Théorèmes 7 et 8 . Il faut cependant prendre des précautions, à cause de l'exemple de [10] qui possède des feuilles exceptionnelles bien que $\pi_{1}(N / \mathscr{G})$ soit isomorphe à $\mathbb{Z}\left[\frac{1}{2}\right]$.

Enfin nous montrerons:

Théorème 15. Si $\pi_{1} N$ est de type fini et n'a pas de quotient libre non abélien, ou bien si $\pi_{1} N$ ne contient pas de sous-groupe libre non abélien et n'a pas de quotient isomorphe à un sous-groupe dense de $\mathbb{Q}$, alors $\mathscr{G} n^{\prime} a$ pas de feuille exceptionnelle.

V. Soit $\omega$ une forme de Morse, comme dans la partie I. En considérant le revêtement galoisien associé au sous-groupe $\mathscr{L} \subset \pi_{1} M$, on construit facilement une action libre de $\pi_{1}(M / \omega)$ sur une variété de dimension 1 , a priori pas séparée, mais simplement connexe. Réciproquement, 
nous montrerons:

Théorème 16. Soit $V$ une variété (non séparée) connexe et simplement connexe, de dimension 1. Soit $G$ un groupe de type fini agissant sur $V$ par $C^{2}$-difféomorphismes, librement et en préservant l'orientation. Alors $G$ est un produit libre de groupes abéliens libres.

L'action est dite libre si $g v=v \Rightarrow g=\mathrm{id}$. Pour montrer le théorème, nous construirons un feuilletage sans holonomie $\mathscr{G}$ sur une variété ouverte $N$, tel que $\pi_{1}(N / \mathscr{G}) \simeq G$. Nous appliquerons alors le Théorème 14 .

Le Théorème 16 est à rapprocher d'une conjecture de Lyndon [14], qui revenait à dire que seul un produit libre de groupes abéliens libres peut agir librement sur un $\mathbb{R}$-arbre (cf. [22]). On sait que cette conjecture est fausse: par exemple, il existe des actions du groupe fondamental d'une surface fermée [16].

En fait, une action libre isométrique sur une 1-variété simplement connexe donne naissance à une action (pas forcément libre) sur un $\mathbb{R}$-arbre. En sens contraire, une action libre sur un $\mathbb{R}$-arbre donne naissance à une action libre isométrique sur une 1-variété (pas forcément simplement connexe) munie d'une métrique riemannienne.

Dans notre contexte, une forme $\omega$ comme ci-dessus donne naissance à une action libre sur un $\mathbb{R}$-arbre, non pas du groupe $\pi_{1} M / \mathscr{L}$, mais du groupe $\pi_{1} M / \overline{\mathscr{L}}$; par $\overline{\mathscr{L}}$, nous désignons le sous-groupe distingué de $\pi_{1} M$ engendré par $\mathscr{L}$ et les classes d'homotopie libre de lacets contenus dans des feuilles sauf en un nombre fini de points qui sont des singularités d'indice 1 ou $n-1$ (voir Figure 1). Pour une forme $\pi_{1}$-stable (voir cidessus résumé de la partie I) on a $\overline{\mathscr{L}}=\mathscr{L}$, mais cette égalité n'est pas toujours vraie.

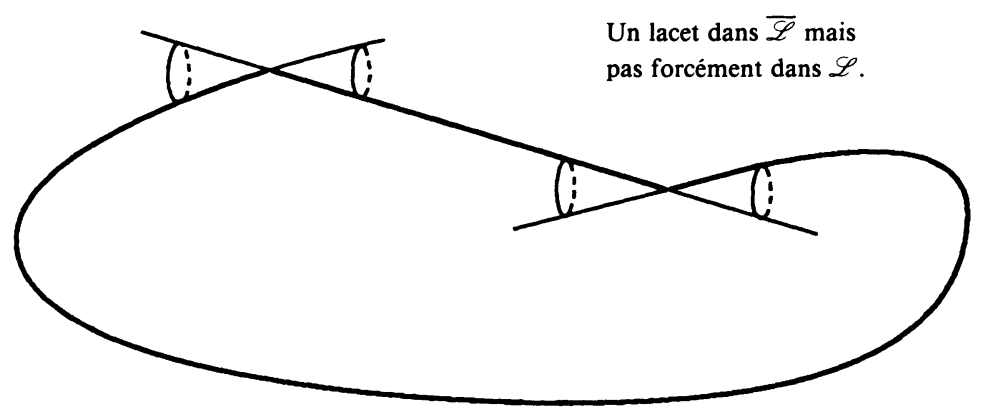

Figure 1

Il conviendrait donc probablement pour étudier les groupes pouvant agir librement sur les $\mathbb{R}$-arbres de généraliser le Théorème 6 dans deux 
directions. D'abord, ne plus supposer le feuilletage transversalement orientable. Ensuite, admettre des singularités de Morse, et ne pas déterminer $\pi_{1} M / \mathscr{L}$ mais $\pi_{1} M / \overline{\mathscr{L}}$.

\section{Préliminaires}

0.A. Groupe fondamental de l'espace des feuilles. Etant donné une 1forme fermée non singulière $\omega$ sur une variété $M$ (pas forcément compacte), nous voulons discuter la notion de groupe fondamental de l'espace des feuilles de $\omega$.

Commençons par un exemple très simple, celui de la forme $\omega_{0}=d y-$ $\alpha d x$ sur le tore $\mathbb{T}^{2}=\mathbb{R}^{2} / \mathbb{Z}^{2}$, avec $\alpha$ irrationnel. En tant qu'ensemble, l'espace des feuillels $\mathbb{T}^{2} / \omega_{0}$ peut être vu comme le quotient d'un cercle de longueur 1 par la rotation $R_{\alpha}$, ou de façon équivalente comme le quotient de $\mathbb{R}$ par le groupe $G$ formé des translations $x \mapsto x+m+n \alpha$, $(m, n) \in \mathbb{Z}^{2} ;$ mais la topologie quotient sur $\mathbb{R} / G$ est la topologie grossière, totalement inutilisable. Il faut donc procéder indirectement pour définir $\pi_{1}\left(\mathbb{T}^{2} / \omega_{0}\right)$.

Le groupe $G$ opère sur l'espace simplement connexe $\mathbb{R}$ de façon libre (seul l'élément neutre de $G$ a des points fixes). Ceci suggère de considérer $\mathbb{R}$ comme le revêtement universel de $\mathbb{T}^{2} / \omega_{0}$, et $G$ comme le groupe de transformations du revêtement. On est ainsi amené à dire que $\pi_{1}\left(\mathbb{T}^{2} / \omega_{0}\right)$ est isomorphe à $G$, c'est à dire à $\mathbb{Z}^{2}$.

On peut également essayer de traiter le feuilletage défini par $\omega_{0}$ comme un fibré (généralisé). Ici les feuilles sont contractiles (difféomorphes à $\mathbb{R}$ ). Il est donc tentant de dire que le $\pi_{1}$ de $\mathbb{T}^{2} / \omega_{0}$ (base du fibré) est isomorphe à celui de $\mathbb{T}^{2}$ (espace total): on retrouve bien $\pi_{1}\left(\mathbb{T}^{2} / \omega_{0}\right) \simeq \mathbb{Z}^{2}$.

Ces deux points de vue peuvent se généraliser au cas d'une forme $\omega$ quelconque. Considérons le revêtement universel $\tilde{M}$ de $M$, et la forme $\tilde{\omega}$ induite par $\omega$. L'espace des feuilles de $\tilde{\omega}$ n'est en général pas séparé, mais c'est une variété $V$ de dimension 1, simplement connexe. Le groupe du revêtement, isomorphe à $\pi_{1} M$, agit sur $V$, mais pas toujours librement: si un élément $\theta \in \pi_{1}\left(\dot{M}, x_{0}\right)$ est représentable par un lacet contenu dans la feuille de $x_{0}$, l'action de $\theta$ sur $V$ aura des points fixes.

Ceci suggère, pour obtenir une action libre, de considérer le plus petit sous-groupe distingué $\mathscr{L} \subset \pi_{1} M$ contentant les images des $\pi_{1}$ des feuilles de $\omega$, le revêtement galoisien $p_{\mathscr{L}}: M_{\mathscr{L}} \rightarrow M$ associé à $\mathscr{L}$, et l'espace des feuilles de $p_{\mathscr{L}}^{*} \omega$, noté $V_{\mathscr{L}}$. On montre que $V_{\mathscr{L}}$ est encore une variété (non séparée) de dimension 1, simplement connexe, et que cette fois le 
groupe de transformations $\pi_{1} M / \mathscr{L}$ agit librement sur $V_{\mathscr{L}}$. On est donc conduit à définir $\pi_{1}(M / \omega)$ comme $\pi_{1} M / \mathscr{L}$.

Dans le cas d'un fibré (à fibre connexe), le $\pi_{1}$ de la base est le quotient du $\pi_{1}$ de l'espace total par l'image du $\pi_{1}$ de la fibre; on a ici un résultat analogue, en remplaçant cette image par le sous-groupe $\mathscr{L}$. $\cap$ n pourrait aussi imaginer, sans changer l'espace des feuilles, de modifier : feuilletage (et la variété $M$ ) en ajoutant des 2-cellules aux feuilles de façon à les rendre simplement connexes; ceci aurait pour effet dans $\pi_{1} M$ de tuer le sous-groupe $\mathscr{L}$, de sorte que le $\pi_{1}$ de l'espace obtenu serait isomorphe à $\pi_{1} M / \mathscr{L}$.

D'une manière plus conceptuelle (et plus précise), on peut, en suivant Haefliger (cf. [7]), associer à un feuilletage quelconque sur une variété $M$ (en fait à son pseudogroupe d'holonomie $\Gamma$ ) un espace classifiant $B \Gamma$. Il faut voir $B \Gamma$ (qui est en général de dimension infinie) comme un espace feuilleté tel que le pseudogroupe d'holonomie soit équivalent à $\Gamma$ et que les revêtements d'holonomie des feuilles soient contractiles.

On définit alors le type d'homotopie et les $\pi_{i}$ de l'espace des feuilles comme ceux de $B \Gamma$, et on montre que $\pi_{1}(B \Gamma)$ est isomorphe au quotient de $\pi_{1} M$ par le sous-groupe distingué engendré par les classes d'homotopie libre des lacets contenus dans des feuilles et à holonomie triviale (voir [20]).

Dans le cas d'un feuilletage défini par une 1-forme fermée $\omega$, il n'y a pas d'holonomie et on retrouve la définition donnée plus haut. On montre d'autre part (Haefliger) que $B \Gamma$ est un $K(\pi, 1)$; il n'y a donc pas d'invariants homotopiques d'ordre supérieur.

L'homomorphisme $[\omega]: \pi_{1} M \rightarrow \mathbb{R}$ obtenu en intégrant la forme le long des lacets se factorise par un homomorphisme $\rho: \pi_{1}(M / \omega) \rightarrow \mathbb{R}$; l'image commune de $[\omega]$ et de $\rho$ est le groupe des périodes $P(\omega)$. On peut en fait considérer $\rho$ comme l'homomorphisme induit au niveau du $\pi_{1}$ par l'application (ensembliste) $\psi: M / \omega \rightarrow \mathbb{R} / P(\omega)$ définie par $\psi(x)=\int_{m}^{x} \omega$, où $m \in M$ est un point base et l'intégrale est regardée modulo $P(\omega)$ (ce qui la rend indépendante du chemin choisi entre $m$ et $x$ ).

Considérons en particulier les formes $\omega$ telles que $\rho$ définisse un isomorphisme entre $\pi_{1}(M / \omega)$ et $P(\omega)$ (nous les appellerons faiblement complètes, généralisant une définition de [13]). Nous avons dit que les espaces des feuilles $M / \omega$ et $\mathbb{R} / P(\omega)$ devaient être vus comme des $K(\pi, 1)$. Ceci conduit à dire qu'une forme est faiblement complète si et seulement si $\psi$ définit une équivalence d'homotopie entre $M / \omega$ et $\mathbb{R} / P(\omega)$.

Terminons en donnant une présentation du groupe fondamental $\pi_{1}(B \Gamma)$ à partir de générateurs d'un pseudogroupe $\Gamma$. Nous nous limitons au cas 
simple où $\Gamma$ est un pseudogroupe défini sur une variété connexe et simplement connexe $W$ (par exemple $W=\mathbb{R}$ immergé dans $M$, transverse à une 1-forme fermée $\omega$, et $\Gamma$ est le pseudogroupe d'holonomie).

Soit $\gamma_{i}: U_{i} \rightarrow V_{i} \quad(i \in I)$ un système de générateurs de $\Gamma$, où chaque $\gamma_{i}$ est un homéomorphisme entre ouverts connexes de $W$. On obtient alors une présentation de $\pi_{1}(B \Gamma)$ en prenant pour générateurs des lettres $a_{i} \quad(i \in I)$, et pour relations les mots en $a_{i}$ et $a_{i}^{-1}$ tels que l'élément correspondant de $\Gamma$ (obtenu en composant les $\gamma_{i}$ ou $\gamma_{i}^{-1}$ ) soit défini et égal à l'identité sur un ouvert non vide de $W$.

0.B. Définitions et notations. Soit $\omega$ une 1-forme fermée sur une variété $M^{n}$. Nous noterons $[\omega]: \pi_{1} M \rightarrow \mathbb{R}$ l'homomorphisme obtenu en intégrant $\omega$ sur les lacets. Son image $P(\omega) \subset \mathbb{R}$ est le groupe des périodes. Le rang de $P(\omega)$, peut-être infini si $M$ n'est pas compacte, sera appelé rang de $\omega$ et noté $r(\omega)$. Rappelons que, si $r(\omega) \leq 1$, alors toute feuille est fermée.

Supposons d'abord $\omega$ non singulière.

0.B.i. Nous noterons $\mathscr{L}(\omega)$, ou simplement $\mathscr{L}$, le plus petit sousgroupe distingué de $\pi_{1} M$ contenant l'image de $\pi_{1} L$ pour toute feuille $L$. Puisque $\mathscr{L}$ est distingué, on peut parler de l'appartenance à $\mathscr{L}$ d'une classe d'homotopie libre de lacets; pour $n \geq 5$, un lacet plongé $\gamma \subset M$ représente un élément de $\mathscr{L}$ si et seulement si $\gamma$ est une composante de $\delta S$, où $S \subset M$ est une surface (plongée) compacte de genre 0 et les autres composantes de $\delta S$ sont contenues dans des feuilles.

Nous aurons parfois à choisir un point base $x \in M$. Le sous-groupe $\mathscr{L} \subset \pi_{1}(M, x)$ est engendré par les lacets de la forme $c \gamma c^{-1}$, où $c$ est un chemin d'origine $x$ et $\gamma$ est un lacet contenu dans une feuille.

Conformément à la section précédente, nous définirons le groupe fondamental de l'espace des feuilles $\pi_{1}(M / \omega)=\pi_{1} M / \mathscr{L}(\omega)$. L'homomorphisme $[\omega]$ se factorise par $\rho: \pi_{1}(M / \omega) \rightarrow \mathbb{R}$. Notons l'isomorphisme $\operatorname{Ker} \rho \simeq$ $\operatorname{Ker}[\omega] / \mathscr{L}(\omega)$.

0.B.ii. Une composante minimale est un ouvert saturé $N \subset M$ tel que toute feuille contenue dans $N$ y soit dense. Noter que $P(\omega \mid N)$ est dense dans $\mathbb{R}$, et que deux composantes minimales sont égales ou disjointes.

Soit $N$ une composante minimale, et $\theta$ la forme induite $\omega \mid N$. On vérifie immédiatement que les conditions suivantes sont équivalentes:

(a) Si un arc $\gamma \subset N$ est d'intégrale nulle $\left(\int_{\gamma} \theta=0\right)$, ses extrémités sont sur la même feuille;

(b) La surjection naturelle $\psi: N / \theta \rightarrow \mathbb{R} / P(\theta)$, obtenue en intégrant $\theta$ à partir d'un point base, est une bijection; 
(c) Il existe une courbe fermée $C$ transverse à $\theta$ telle que, si $x$ et $y$ bordent sur $C$ un arc $I$ avec $\int_{I} \theta \in P(\theta)$, alors $x$ et $y$ sont sur la même feuille.

Si ces conditions sont vérifiées, nous dirons que $(N, \theta)$ est une composante faiblement complète. On notera qu'alors toute courbe transverse possède la propriété énoncée dans $c$, et que toute période d $\theta$ (sauf 0 ) peut être représentée par une courbe transverse. Nous montrerons (I.A et II.B) que $(N, \theta)$ est faiblement complète si et seulement si $\pi_{1}(N / \theta) \simeq$ $P(\theta)$.

Soit $C$ une courbe transverse dans une composante faiblement complète $(N, \theta)$. La forme non singulière induite sur $C$ par $\theta$ définit sur $C$ une mesure de Lebesgue; en particulier, on peut parler de rotations sur $C$.

La condition $c$ ci-dessus signifie en fait que le pseudogroupe d'holonomie de $\theta$ sur $C$ est précisément le pseudogroupe engendré par le groupe $P(\theta)$ agissant par rotations; en d'autres termes, les intersections avec $C$ des feuilles de $\theta$ sont les orbites de l'action de $P(\theta)$.

On notera que les pseudogroupes ainsi engendrés par un groupe de rotations sont ceux qui sont complets au sens de [7]. Ils sont caractérisés par la propriété suivante: si $R$ est une rotation de $C$ et si pour un $x \in C$ les points $x$ et $R(x)$ sont sur la même feuille, alors il en est de même pour tout $x \in C$.

Nous utiliserons dans II.B la remarque suivante. Soit $(N, \theta)$ une composante minimale, et $C$ une courbe transverse; s'il existe un groupe dense $G$ de rotations de $C$ tel que $x$ et $R(x)$ soient sur la même feuille pour tout $x \in C$ et tout $R \in G$, alors $(N, \theta)$ est faiblement complète.

En effet, d'après le Lemme III.5 de [13], l'holonomie de $\theta$ sur $C$ est définie par l'action d'un groupe de rotations. Pour voir que ce groupe est $P(\theta)$ tout entier, il suffit de représenter une période par un lacet $\gamma:[0,1] \rightarrow N$, et de subdiviser $[0,1]$ par $0=t_{0}<\cdots<t_{k}=1$ de façon que les $\gamma\left(t_{i}\right)$ soient tous sur la même feuille $L$, et que chaque $\gamma\left(t_{i}\right)$ soit joignable dans $L$ à un point de $C$ par un chemin relevable continûment sur les feuilles voisines jusqu'à $\gamma\left(t_{i+1}\right)$.

0.B.iii. Si maintenant $\omega$ a des singularités (i.e., des points $x$ où $\omega_{x}=$ 0 ), nous noterons $M^{*}=M-\operatorname{Sing} \omega$ et $\omega^{*}$ la forme induite sur $M^{*}$. Par définition, les feuilles, composantes minimales, composantes faiblement complètes de $\omega$ seront celles de $\omega^{*}$. Une feuille non compacte $L$ est presque compacte si $L \cup \operatorname{Sing} \omega$ est compact.

Nous supposerons toujours que $M^{*}$ est connexe et que l'inclusion induit un isomorphisme $\pi_{1} M^{*} \simeq \pi_{1} M$; ce sera en particulier le cas si Sing $\omega$ est 
de codimension $\geq 3$. Nous définissons $\mathscr{L}(\omega)=\mathscr{L}\left(\omega^{*}\right)$ et $\pi_{1}(M / \omega)=$ $\pi_{1} M / \mathscr{L}(\omega)$ comme précédemment.

Supposons désormais que $M$ est compacte, orientable, sans bord, de dimension $n \geq 3$, et que $\omega$ est de Morse: au voisinage de chaque singularité, $\omega$ est la différentielle d'une fonction de Morse. Les singularités ont ainsi un indice, compris entre 0 et $n$.

Celles d'indice 0 ou $n$ sont des centres; les feuilles au voisinage sont difféomorphes à des sphères. Celles d'indice 1 ou $n-1$ sont dites coniques (cf. Figure 1 ou 2); il en part deux bouts singuliers (demi-cônes). Si ces deux bouts singuliers ne sont pas contenus dans la même feuille (i.e., s'il n'existe pas de lacet de connexion au sens de [13]), la singularité est bloquante.
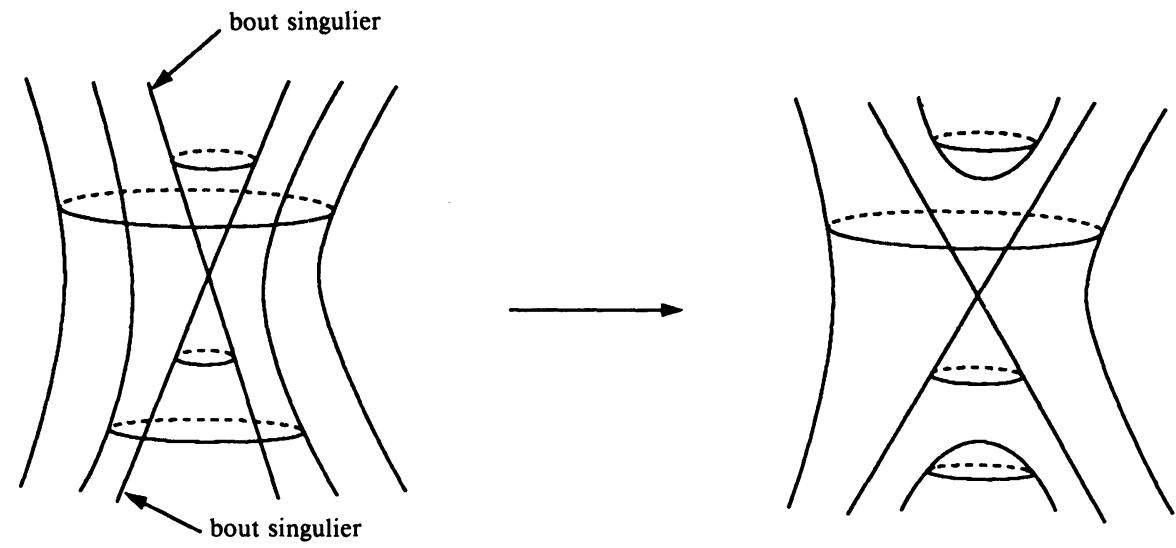

Figure 2: COUPURE DE FEUILLES AU VOISINAGE D'UNE SINGULARITÉ CONIQUE

0.B.iv. La présence de singularités bloquantes correspond au fait que le pseudogroupe d'holonomie induit par $\omega$ sur des courbes transverses n'est pas défini par l'action d'un groupe de rotations. D'après [13, Proposition II.1], les formes de rang $\geq 2$ sans singularité bloquante sont précisément celles qui sont minimales et faiblement complètes (i.e., $\left(M^{*}, \omega^{*}\right)$ est une composante minimale faiblement complète).

Les composantes minimales de $\omega$ sont en nombre fini. On montre ([9]; voir l'appendice de [1]) qu'une feuille $L$ n'appartenant pas à une composante minimale est compacte ou presque compacte. On notera que les feuilles presque compactes sont en nombre fini.

0.C. Produits libres de groupes abéliens libres (proligals). Soit $G$ un groupe de type fini. Nous noterons $b(G)$ le rang maximal d'un quotient 
abélien libre de $G$, et $f(G)$ le rang maximal d'un quotient libre de $G$. Bien sûr $b(G) \geq f(G)$. Si d'autre part $G^{\prime}$ est un quotient de $G$, alors $b(G) \geq b\left(G^{\prime}\right)$ et $f(G) \geq f\left(G^{\prime}\right)$.

Lorsque $G$ est le $\pi_{1}$ d'une variété $M$, nous noterons $b_{1} M=b(G)$ et $b_{1}^{\prime} M=f(G)$ (premiers nombres de Betti, usuel et non commutatif). Si $G=\pi_{1}(M / \omega)$, nous poserons $b(\omega)=b(G)$ et $d(\omega)=f(G)-1$.

Nous dirons que $G$ est un proligal (PROduit LIbre de Groupes Abéliens Libres) s'il est isomorphe à un produit libre $\mathbb{Z}^{a_{1}} * \cdots * \mathbb{Z}^{a_{f}}, a_{1} \geq \cdots \geq a_{f} \geq$ 1. Un tel isomorphisme n'est pas unique, mais $f$ et les $a_{i}$ sont bien déterminés [15]. Notons que $f=f(G)$ et $\sum a_{i}=b(G)$.

Nous utiliserons la conséquence suivante du théorème de Kurosh [15]: tout sous-groupe abélien non cyclique d'un proligal est conjugué à un sousgroupe d'un facteur $\mathbb{Z}^{a_{i}}\left(a_{i} \geq 2\right)$. En particulier, les facteurs $\mathbb{Z}^{a_{i}}$ de rang $a_{i} \geq 2$ sont bien déterminés à conjugaison près: ce sont les sous-groupes abéliens maximaux non cycliques.

Lemme 0. Soit $\varphi: G \rightarrow G^{\prime}$ un épimorphisme. On suppose que $G$ et $G^{\prime}$ sont des proligals et que $\varphi$ est injectif sur chaque facteur de $G$ de rang $a_{i} \geq 2$. Alors ou bien $\varphi$ est un isomorphisme ou bien $f(G)>f\left(G^{\prime}\right)$.

Remarque. Si on ne suppose pas l'injectivité de $\varphi$ sur les facteurs de rang $\geq 2$, on peut montrer que $\varphi$ est un isomorphisme pourvu que $f(G)=f\left(G^{\prime}\right)$ et $b(G)=b\left(G^{\prime}\right)$.

Démonstration. Nous supposons $f(G)=f\left(G^{\prime}\right)=f$, et nous allons montrer que $\varphi$ est un isomorphisme. Il suffit de voir que $G$ et $G^{\prime}$ sont isomorphes abstraitement (i.e., que les $a_{i}$ associés à $G$ coïncident avec les $a_{i}^{\prime}$ associés à $G^{\prime}$ ), car d'après [4] un proligal $G$ est hopfien: tout épimorphisme $G \rightarrow G$ est un isomorphisme (un proligal est même résiduellement fini [5]).

D'après le théorème de Kurosh, l'image par $\varphi$ d'un facteur $\mathbb{Z}^{a_{i}}$ de rang $\geq 2$ est contenue dans un conjugué d'un facteur $\mathbb{Z}^{a_{\sigma(i)}^{\prime}}$ de $G^{\prime}$. Appelons $k$ le plus grand indice pour lequel $a_{k} \geq 2$, et considérons l'application $\sigma:\{1, \cdots, k\} \rightarrow\{1, \cdots, f\}$.

Admettons provisoirement que $\sigma$ est injective. Nous avons alors

$$
\begin{aligned}
b(G)= & \sum_{i=1}^{f} a_{i}=f+\sum_{i=1}^{k}\left(a_{i}-1\right) \leq f+\sum_{i=1}^{k}\left(a_{\sigma(i)}^{\prime}-1\right) \\
& \leq f+\sum_{j=1}^{f}\left(a_{j}^{\prime}-1\right)=b\left(G^{\prime}\right) \leq b(G) .
\end{aligned}
$$

Toutes ces inégalités sont ainsi des égalités, et on en déduit facilement le résultat. 
Supposons donc qu'il existe $p$ et $q$ dans $\{1, \cdots, k\}$ avec $\sigma(p)=$ $\sigma(q)$, et cherchons une contradiction. Considérons un épimorphisme $\psi: G^{\prime} \rightarrow \mathbb{Z}^{f}=\prod_{i=1}^{f} \mathbb{Z}$ envoyant chaque facteur $\mathbb{Z}^{a_{i}^{\prime}}$ de $G^{\prime}$ surjectivement sur le facteur correspondant de $\mathbb{Z}^{f}$. Le composé $\theta=\psi \circ \varphi: G \rightarrow \mathbb{Z}^{f}$ envoie $\mathbb{Z}^{a_{p}}$ et $\mathbb{Z}^{a_{q}}$ dans le même facteur $\psi\left(\mathbb{Z}^{a_{\sigma(p)}^{\prime}}\right)$ de $\mathbb{Z}^{f}$.

Remarquons que chaque $\theta\left(\mathbb{Z}^{a_{i}}\right)$ est isomorphe à $\{0\}$ ou $\mathbb{Z}$, et factorisons $\theta$ par le groupe libre $F=*_{i=1}^{f} \theta\left(\mathbb{Z}^{a_{i}}\right)$. Puisque $F$ admet $\mathbb{Z}^{f}$ comme quotient, il doit être de rang $f$. En particulier aucun $\theta\left(\mathbb{Z}^{a_{i}}\right)$ n'est réduit à $\{0\}$, et $\theta$ induit un isomorphisme entre l'abélianisé $F_{\text {ab }}$ et $\mathbb{Z}^{f}$. On en déduit que le sous-groupe de $\mathbb{Z}^{f}$ engendré par l'union de $\theta\left(\mathbb{Z}^{a_{p}}\right)$ et $\theta\left(\mathbb{Z}^{a_{a}}\right)$ est de rang 2 , ce qui est la contradiction cherchée.

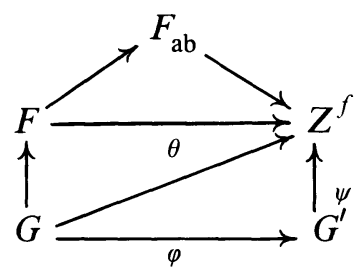

(Tous les morphismes de ce diagramme commutatif sont surjectifs.)

\section{Formes de Morse sur les variétés fermées}

Dans toute cette partie, nous appellerons $\omega$ une 1-forme fermée de Morse sur une variété fermée orientable $M^{n} \quad(n \geq 3)$.

I.A. Détermination de $\pi_{1}(M / \omega)$.

Théorème I.1. Soit $M^{n}$ une variété fermée orientable de dimension $n \geq 3$, et $\omega$ une 1-forme fermée de Morse sur $M$. Alors $\pi_{1}(M / \omega)$ est un produit libre de groupes abéliens libres. De plus, les facteurs de rang $\geq 2$ sont en bijection avec les groupes des périodes des composantes minimales faiblement complètes de $\omega$.

Si on appelle $U_{1}, \cdots, U_{m}$ les composantes faiblement complètes de $\omega$, la deuxième assertion du théorème signifie que $\pi_{1}(M / \omega)$ est isomorphe à $F * P_{1} * \cdots * P_{m}$, où $F$ est libre et $\rho \mid P_{i}$ définit un isomorphisme de $P_{i}$ sur $P\left(\omega \mid U_{i}\right)$.

Définition. Nous noterons $d(\omega)$ le nombre de facteurs du produit libre $\pi_{1}(M / \omega)$, diminué de 1 .

Remarques. (1) Il résultera de la partie II.B que le Théorème I.1 reste vrai si:

(i) $M$ n'est pas forcément compacte, mais $\pi_{1} M$ est de type fini; 
(ii) Les singularités de $\omega$ sont quelconques, mais l'inclusion $M^{*} \subset M$ induit un isomorphisme des $\pi_{1}$ (en particulier si Sing $\omega$ est de codimension $\geq 3$ ).

(2) $\mathrm{Si} M$ est une surface, on peut montrer que $\pi_{1}\left(M^{*} / \omega^{*}\right)$ est libre, sauf si $\omega$ est non singulière de rang 2 sur $\mathbb{T}^{2}$.

Notons deux conséquences du Théorème I.1 avant de le montrer.

Corollaire. Si $\omega$ n'a pas de composante faiblement complète, alors $\pi_{1}(M / \omega)$ est libre, et réciproquement.

Corollaire. Si $\omega$ n'est pas exacte, alors $\operatorname{Ker} \rho \simeq \operatorname{Ker}[\omega] / \mathscr{L}(\omega)$ est soit égal à $\{1\}$, soit libre à une infinité de générateurs.

Démonstration. D'après la deuxième assertion du Théorème I.1, Ker $\rho$ rencontre trivialement tout conjugué d'un facteur de rang $\geq 2$ de $\pi_{1}(M / \omega)$. Il est donc libre d'après le théorème de Kurosh [15]. Si $\rho$ n'est pas injective et si $\omega$ n'est pas exacte, alors $\operatorname{Ker} \rho \subset \pi_{1}(M / \omega)$ est un sous-groupe distingué non trivial d'indice infini dans un produit libre non trivial. Il ne peut donc pas être de type fini d'après le Lemme 11.2 de [8].

Démonstration $d u$ Théorème I.1. Nous procédons en trois étapes. L'étape importante est la troisième, où sont traitées les composantes minimales non faiblement complètes.

(1) Déterminons d'abord le $\pi_{1}$ de l'espace des feuilles d'une composante faiblement complète.

Proposition 1.2. Soit $\alpha$ une 1-forme fermée non singulière sur une variété $N . S i(N, \alpha)$ est une composante faiblement complète, alors l'épimorphisme $\rho: \pi_{1}(N / \alpha) \rightarrow P(\alpha)$ est un isomorphisme.

Remarques. -Des cas particuliers de ce résultat ont été montrés dans [13].

-Cette proposition peut s'interpréter de la façon suivante: puisque $\alpha$ est faiblement complète, son pseudogroupe d'holonomie $\Gamma$ est équivalent au pseudogroupe engendré par le groupe $G=P(\alpha)$ agissant sur $\mathbb{R}$ par translations; pour un tel pseudogroupe, on a $\pi_{1}(B \Gamma) \simeq G$.

Démonstration. Commençons par une remarque. Soit $X$ un champ de vecteurs sur $N$ tel que $\alpha(X) \equiv 1$, et $\varphi_{t}$ son flot (partiellement défini). Soit $\sigma$ un chemin contenu dans une feuille, et $\tau \in \mathbb{R}$ tel que $\varphi_{\tau}$ soit défini aux deux extrémités $a$ et $b$ de $\sigma$.

Pour $t$ entre 0 et $\tau$, les points $\varphi_{t}(a)$ et $\varphi_{t}(b)$ appartiennent à la même feuille $L_{t}$ (car $\alpha$ est faiblement complète); nous pouvons donc considérer un lacet $\beta_{t}$ obtenu en allant de $a$ à $b$ par $\sigma$, de $b$ à $\varphi_{t}(b)$ en suivant l'orbite de $X$, de $\varphi_{t}(b)$ à $\varphi_{t}(a)$ par un chemin $\gamma_{t}$ dans $L_{t}$, et en revenant de $\varphi_{t}(a)$ à $a$. 
Le fait que $\beta_{t}$ représente ou non un élément de $\mathscr{L}$ ne dépend pas du choix de $\gamma_{t}$ dans $L_{t}$, et on vérifie immédiatement que l'ensemble des $t$ tels que $\beta_{t} \in \mathscr{L}$ est ouvert et fermé, donc contient $\tau$.

Ceci noté, considérons les lacets $C^{\infty}$ par morceaux formés de segments $\lambda_{i}$ transverses à $\alpha$ et de segments $\sigma_{i}$ contenus dans des feuilles. Si la proposition est fausse, il existe des lacets de ce type dont la classe d'homotopie libre est dans $\operatorname{Ker}[\alpha]$, mais pas dans $\mathscr{L}$; fixons un tel $\gamma$ pour lequel le nombre de $\lambda_{i}$ est minimal.

Construisons (par partition de l'unité) un champ $X$ avec $\alpha(X) \equiv 1$, tel que les $\lambda_{i}$ soient des morceaux d'orbite. Grâce à la remarque ci-dessus, on peut modifier $\gamma$ par un élément de $\mathscr{L}$, de façon à diminuer le nombre des $\lambda_{i}$ : contradiction. q.e.d.

(2) Montrons maintenant le Théorème I.1 dans le cas où toutes les composantes minimales de $\omega$ sont faiblement complètes. Nous faisons de plus une hypothèse technique: il n'existe pas de chemin $\left[s, s^{\prime}\right]$ tel que $] s, s^{\prime}[$ est contenu dans une feuille, $s$ est une singularité d'indice 1 , et $s^{\prime}$ est une singularité d'indice $n-1$ (absence de liaison entre singularités d'indices 1 et $n-1)$.

Les composantes $W_{k}$ de l'ouvert formé des feuilles compactes de $\omega$ peuvent être vues comme des intervalles, car elles sont feuilletées comme des produits: feuille compacte $\times$ intervalle ouvert. A chaque bout de $W_{k}$, la feuille compacte dégénère en une union de singularités de $\omega$ et de feuilles presque compactes.

Chaque feuille contenue dans la frontière d'une composante minimale $U_{i}$ est presque compacte et adhère d'un côté à $U_{i}$ et de l'autre à un $W_{k}$ (ceci utilise l'absence de liaison $1 / n-1$ ); en particulier, les $\bar{U}_{i}$ sont deux à deux disjoints.

Considérons l'ensemble des feuilles non compactes de $\omega$, muni de la relation d'équivalence que l'on engendre en identifiant deux feuilles $L$ et $L^{\prime}$ si $\bar{L} \cap \bar{L}^{\prime} \neq \varnothing$. Une classe d'équivalence se compose soit de toutes les feuilles contenues dans l'adhérence d'une composante minimale $U_{i}$, soit de feuilles presque compactes.

Nous allons maintenant associer à $\omega$ un graphe fini orienté $T_{\omega} . \mathrm{Si}$ $\omega$ n'a pas de composante minimale, ce sera le graphe obtenu en écrasant chaque feuille en un point. En général, il faudra au préalable écraser en un point chaque composante minimale.

Par définition, les sommets de $T_{\omega}$ seront d'une part les centres de $\omega$, d'autre part les classes de la relation ci-dessus. Les arêtes seront les $W_{k}$, vus comme des intervalles orientés par l'orientation transverse du feuilletage, et attachés aux sommets de la façon évidente. 
Intuitivement, l'espace des feuilles de $\omega$ peut être construit à partir de $T_{\omega}$ en attachant les espaces des feuilles des $\omega \mid U_{i}$ aux sommets correspondants. Son $\pi_{1}$ doit donc être le produit libre du groupe libre $F=\pi_{1}\left(T_{\omega}\right)$ avec les $\pi_{1}\left(U_{i} / \omega\right)$, qui sont isomorphes à $P\left(\omega \mid U_{i}\right)$ d'après la Proposition I.2. C'est ce que nous allons maintenant vérifier en construisant un isomorphisme

$$
\pi_{1}(M / \omega) \rightarrow F * P\left(\omega \mid U_{1}\right) * \cdots * P\left(\omega \mid U_{m}\right) .
$$

Remarque. Deux feuilles presque compactes différentes contenant chacune un bout singulier d'une même singularité bloquante sont représentées par le même point de $T_{\omega}$. En toute rigueur, il faudrait donc remplacer le graphe $T_{\omega}$ par une variété non séparée de dimension 1. Toutefois, l'absence de liaison entre singularités d'indice 1 et $n-1$ fait que cette inexactitude est sans influence sur le $\pi_{1}$.

Choisissons un arbre maximal dans $T_{\omega}$, et une feuille compacte $L_{\alpha}$ sur chaque arête du complémentaire. Le groupe $F$ peut être vu comme le groupe libre engendré par les lettres $L_{\alpha}$.

Pour chaque composante minimale $U_{i}$ et chaque feuille $L_{i, j}$ de la frontière de $U_{i}$, choisissons un point base $u_{i} \in U_{i}$, un chemin allant de $u_{i}$ à $L_{i, j}$ dans $U_{i}$, et appelons $c_{i, j}$ l'intégrale de $\omega$ sur ce chemin. Choisissons enfin dans $M$ un point base $x$ appartenant à une feuille compacte autre qu'une $L_{c k}$ (s'il n'y a pas de feuille compacte, la Proposition I. 2 donne le résultat).

Définissons un homomorphisme

$$
\theta: \pi_{1}(M, x) \rightarrow F * P\left(\omega \mid U_{1}\right) * \cdots * P\left(\omega \mid U_{m}\right) .
$$

Soit $\gamma$ un lacet disjoint de Sing $\omega$ et transverse aux $L_{\alpha}$ et aux $L_{i, j}$. Pour définir $\theta(\gamma)$, parcourons $\gamma$. Lorsqu'on rencontre une feuille $L_{\alpha}$, on écrit la lettre $L_{\alpha}$ ou $\left(L_{\alpha}\right)^{-1}$ de $F$, selon que l'orientation de $\gamma$ coïncide ou non avec l'orientation transverse de $L_{\alpha}$. Lorsqu'on traverse une composante minimale $U_{i}$, en entrant par un $L_{i, j}$ et en sortant par un $L_{i, j^{\prime}}$, on écrit l'élément $c+c_{i, j}-c_{i, j^{\prime}}$ du facteur $P\left(\omega \mid U_{i}\right)$, où $c$ est l'intégrale de $\omega$ sur l'arc de $\gamma$ allant de $L_{i, j}$ à $L_{i, j^{\prime}}$.

Il est clair que $\theta(\gamma)$ ne dépend que de la classe d'homotopie de $\gamma$, que $\theta$ est surjectif, et que $\operatorname{Ker} \theta$ contient $\mathscr{L}$. Il nous reste à vérifier que $\operatorname{Ker} \theta$ ne contient rien d'autre.

Si tel n'est pas le cas, considérons les lacets basés en $x$, disjoints de Sing $\omega$, représentant un élément de $\operatorname{Ker} \theta-\mathscr{L}$, et choisissons un tel $\gamma$ pour lequel le nombre $i_{\gamma}$ de points d'intersection avec l'union des feuilles presque compactes est minimal. On a $i_{\gamma}>0$, car sinon $\gamma$ est contenu dans un $W_{k}$ et représente un élément de $\mathscr{L}$. 
Puisque $\gamma \in \operatorname{Ker} \theta$, il existe une feuille presque compacte $L$ et un sousarc $\gamma^{\prime}$ de $\gamma$, à extrémités sur $L$, d'intérieur disjoint des feuilles presque compactes, avec $\int_{\gamma^{\prime}} \omega=0$. Si l'intérieur de $\gamma^{\prime}$ est contenu dans un $W_{k}$, on peut modifier $\gamma$ par un élément de $\mathscr{L}$ pour diminuer $i_{\gamma}$ de 2 . Sinon, l'intérieur de $\gamma^{\prime}$ est contenu dans une composante faiblement complète, et la Proposition I. 2 permet encore de diminuer $i_{\gamma}$.

(3) La démonstration dans le cas général repose d'une part sur le Lemme 0 (voir $§ 0 . C$ ci-dessus), d'autre part sur une idée de perturbation introduite dans [13].

Pour chaque singularité $s$ d'indice $n-1$, choisissons une "fonction bosse" $f_{s}: M \rightarrow[0,1]$, de classe $C^{\infty}$, égale à 1 sur un petit voisinage de $s$ et à 0 en dehors d'un voisinage un peu plus grand. Approchons $\omega$ par des formes $\omega(\varepsilon)=\omega-\sum_{s} \varepsilon \cdot d f_{s}$, où $\varepsilon$ est un réel strictement positif.

Les $\omega(\varepsilon)$ sont cohomologues à $\omega$ et, pour $\varepsilon$ petit, ont les mêmes singularités que $\omega$. Notons que le passage de $\omega$ à $\omega(\varepsilon)$ se fait en coupant des feuilles près des singularités d'indice $n-1$ (cf. [13, I.7.2] et Figure $2)$. En particulier, $\mathscr{L}(\omega)$ est l'union croissante des $\mathscr{L}(\omega(\varepsilon))$ quand $\varepsilon$ décroît vers 0 .

Nous avons montré dans [13, pp. 651-652] que, si $\omega$ est minimale mais pas faiblement complète, alors toutes les feuilles régulières de $\omega(\varepsilon)$ sont compactes. Le même raisonnement (basé sur le Lemme III.5) montre que les composantes minimales non faiblement complètes de $\omega$ disparaissent dans $\omega(\varepsilon)$ : elles sont remplacées par des feuilles compactes.

Par contre (cf. [13, I.7.3 et II]), la forme $\omega(\varepsilon)$ est minimale et faiblement complète si $\omega$ l'est (pour $\varepsilon$ assez petit). Plus généralement, les composantes faiblement complètes de $\omega$ subsistent dans $\omega(\varepsilon)$, avec le même groupe des périodes.

Choisissons une suite $\varepsilon_{p}$ tendant vers 0 de façon monotone, et posons $\omega_{p}=\omega\left(\varepsilon_{p}\right)$. Nous pouvons supposer sans perte de généralité que les $\omega_{p}$ n'ont pas de liaison $1 / n-1$ : deux singularités $s$ et $s^{\prime}$ d'indices respectifs 1 et $n-1$ ne peuvent être reliées dans $\omega_{p}$ que pour une seule valeur de $\varepsilon_{p}$ modulo $P(\omega)$.

Pour $p$ assez grand, les formes $\omega_{p}$ vérifient les hypothèses de l'étape 2. Les $\pi_{1}\left(M / \omega_{p}\right)$ sont donc des proligals. Appliquons le Lemme 0 aux épimorphismes $\pi_{1}\left(M / \omega_{p}\right) \rightarrow \pi_{1}\left(M / \omega_{p+1}\right)$ induits par les inclusions $\mathscr{L}\left(\omega_{p}\right) \subset \mathscr{L}\left(\omega_{p+1}\right)$. Pour $p$ assez grand, la suite $\mathscr{L}\left(\omega_{p}\right)$ doit être stationnaire. Donc $\mathscr{L}(\omega)=\mathscr{L}\left(\omega_{p}\right)$ pour $p$ grand, d'où le résultat.

Remarque I.3. Ce raisonnement montre que, pour $\varepsilon$ assez petit, les formes $\omega$ et $\omega(\varepsilon)$ ont le même $\mathscr{L}$. Plus généralement, prenons une 
fonction bosse $f_{s}$ près de chaque singularité $s$ de $\omega$. Soit $\omega^{\prime}=\omega-$ $\sum_{s} \varepsilon(s) \cdot d f_{s}$, avec $\varepsilon(s)>0$ si $s$ est d'indice $n-1$ et $\varepsilon(s) \leq 0$ si $s$ est d'indice 1 . Si les $\varepsilon(s)$ sont assez proches de 0 , alors toutes les composantes minimales de $\omega^{\prime}$ sont faiblement complètes et $\mathscr{L}\left(\omega^{\prime}\right)=\mathscr{L}(\omega)$. Notons de plus que, pour presque tout choix des $\varepsilon(s)$, la forme $\omega^{\prime}$ n'a aucune liaison entre singularités.

I.B. Formes $\pi_{1}$-stables. Etant donné $\Omega \in H^{1}(M, \mathbb{R})$, soit $F(\Omega)$ l'espace des 1 -formes fermées représentant $\Omega$, muni de la topologie $C^{r}$ (avec $1 \leq r \leq \infty)$. Dans $F(\Omega)$, les formes de Morse constituent un ouvert dense $F_{m}(\Omega)$.

Si $\omega$ est de Morse, alors $\pi_{1}(M / \omega)$ est de présentation finie d'après le Théorème I.1. Il existe donc un nombre fini de lacets $\gamma_{i}$ contenus dans des feuilles de $\omega$, tels que $\mathscr{L}(\omega)$ soit le plus petit sous-groupe distingué de $\pi_{1} M$ contenant les classes d'homotopie libre des $\gamma_{i}$.

Pour $\omega^{\prime}$ cohomologue à $\omega$ et proche de $\omega$, il y aura, au voisinage de chaque $\gamma_{i}$, un lacet $\gamma_{i}^{\prime}$ contenu dans une feuille de $\omega^{\prime}$. En particulier, $\mathscr{L}(\omega) \subset \mathscr{L}\left(\omega^{\prime}\right)$. Donc:

Proposition 1.4. Soit $\omega$ une forme de Morse, et $\Omega$ sa classe de cohomologie. Si $\omega^{\prime}$ est proche de $\omega$ dans $F(\Omega)$, on a $\mathscr{L}(\omega) \subset \mathscr{L}\left(\omega^{\prime}\right)$, et donc $d(\omega) \geq d\left(\omega^{\prime}\right)$. En particulier, la fonction $d$ est semi-continue supérieurement sur chaque $F_{m}(\Omega)$.

Disons qu'une forme de Morse $\omega$ est $\pi_{1}$-stable si $\pi_{1}(M / \omega)$ reste inchangé lorsqu'on perturbe $\omega$ dans sa classe de cohomologie, i.e. s'il existe un voisinage $U$ de $\omega$ dans $F(\Omega)$ tel que $\omega^{\prime} \in U \Rightarrow \mathscr{L}\left(\omega^{\prime}\right)=$ $\mathscr{L}(\omega)$.

Proposition I.5. (1) Une forme de Morse $\omega$ est $\pi_{1}$-stable si et seulement si $d\left(\omega^{\prime}\right)=d(\omega)$ pour toute $\omega^{\prime}$ proche de $\omega$ dans $F(\Omega)$.

(2) Dans $F(\Omega)$, l'ensemble des formes de Morse $\pi_{1}$-stables est un ouvert dense.

(3) Si $\omega$ est $\pi_{1}$-stable, toutes ses composantes minimales sont faiblement complètes.

Remarque. On peut également montrer que, dans une forme $\pi_{1}$-stable, les composantes minimales ont des adhérences disjointes.

Démonstration. Le point 1 résulte immédiatement du Lemme 0. Pour la densité dans 2 , soit $U$ un ouvert non vide de $F_{m}(\Omega)$. Toute forme de $U$ réalisant le minimum de $d$ dans $U$ est $\pi_{1}$-stable. Enfin, si $\omega$ possède une composante minimale non faiblement complète, on peut perturber $\omega$ dans $F(\Omega)$ de façon à rendre cette composante faiblement complète [13, pp. 651-652]. Ceci change $\pi_{1}(M / \omega)$. q.e.d. 
La Proposition I.5 entraîne que génériquement, toutes les composantes minimales sont faiblement complètes. Plus précisément, soit $F_{M}$ l'union des $F(\Omega)$, c'est-à-dire l'espace de toutes les formes fermées sur $M$. Alors:

Théorème I.6. L'ensemble des formes de Morse dont toutes les composantes minimales sont faiblement complètes contient:

-un ouvert dense dans chaque $F(\Omega)$;

-un $G_{\delta}$ dense dans $F_{M}$.

Remarque. La première assertion est évidemment triviale si $\Omega$ est de rang $\leq 1$ (car toute forme représentant $\Omega$ est à feuilles régulières compactes).

Démonstration. La première assertion résulte immédiatement de la Proposition I.5. Pour la deuxième, rappelons qu'une forme est totalement irrationnelle si son rang est égal au premier nombre de Betti $b_{1} M$ (cf. [13, I.1]). Considérons l'ensemble $D$ des formes de Morse totalement irrationnelles $\omega$ telles que, si $\omega^{\prime}$ est totalement irrationnelle et proche de $\omega$, alors $d\left(\omega^{\prime}\right)=d(\omega)$.

Une forme $\omega \in D$ est en particulier $\pi_{1}$-stable, donc à composantes minimales faiblement complètes. D'autre part, $D$ est ouvert dans le $G_{\delta}$ dense des formes totalement irrationnelles. Il ne reste donc plus qu'à vérifier que $D$ est dense dans $F_{M}$.

Soit $\omega$ de Morse totalement irrationnelle, et $\gamma_{i}$ un nombre fini de lacets engendrant $\mathscr{L}(\omega)$ en tant que sous-groupe distingué, comme plus haut. Puisque $\omega$ est totalement irrationnelle, l'intégrale sur $\gamma_{i}$ de n'importe quelle forme est nulle. En particulier, on aura $\mathscr{L}(\omega) \subset \mathscr{L}\left(\omega^{\prime}\right)$ pour toute forme $\omega^{\prime}$ proche de $\omega$. On en déduit que la restriction de $d$ à l'ensemble des formes de Morse totalement irrationnelles est semi-continue supérieurement, donc continue en un ensemble dense de points.

I.C. Invariant $d$ et singularités coniques.

I.C.i. Calcul de $d(\omega)$. Nous avons associé à une forme de Morse $\omega$ un entier $d=d(\omega)$, en disant que $\pi_{1}(M / \omega)$ est le produit libre de $d+1$ facteurs. Nous allons maintenant interpréter $d$ géométriquement, en fonction des singularités bloquantes (cf. O.B.iii) de $\omega$.

Pour les formes sans centre, $d$ est le nombre de singularités bloquantes d'indice 1 (resp. $n-1$ ), diminué d'un terme correctif (nul génériquement) dû à d'éventuels cycles de liaisons entre singularités bloquantes. Si $\omega$ possède des centres, il faut également retrancher le nombre de singularités d'indice 0 (resp. $n$ ).

Plus précisément, appelons $c_{i}$ le nombre de singularités d'indice $i$ de $\omega$, et $c_{1, b}$ (resp. $c_{n-1, b}$ ) le nombre de singularités bloquantes d'indice 1 (resp. $n-1)$. 
Définissons d'autre part un 1-complexe fini $K$. Les sommets sont les feuilles singulières contenant au moins un bout singulier d'indice 1 (i.e. issu d'une singularité d'indice 1); les arêtes sont les singularités d'indice 1, attachées aux sommets de la façon évidente. Les singularités bloquantes d'indice 1 sont précisément les arêtes à extrémités distinctes.

Appelons $\bar{c}_{1, b}$ le nombre de sommets de $K$ moins le nombre de composantes connexes, c'est-à-dire $\bar{c}_{1, b}=\sum\left(v_{q}-1\right)$ où $v_{q}$ est le nombre de sommets de la composante $K_{q}$ de $K$. Bien sûr $\bar{c}_{1, b} \leq c_{1, b}$, avec égalité dans la situation (générique) où il n'y a pas de liaison entre singularités d'indice 1. Définissons de même $\bar{c}_{n-1, b}$.

Théorème I.7. Soit $\omega$ une forme de Morse. Alors $d=\bar{c}_{1, b}-c_{0}=$ $\bar{c}_{n-1, b}-c_{n}$.

Corollaire. On a toujours $c_{1, b} \geq d+c_{0}$ et $c_{n-1, b} \geq d+c_{n}$, avec égalité génériquement dans chaque $F_{m}(\Omega)$.

Remarque. On a $\bar{c}_{1, b}=\bar{c}_{n-1, b}=0$ si et seulement si $c_{1, b}=c_{n-1, b}=$ 0 . Pour $\omega$ sans centre, $d=0$ équivaut donc à l'absence de singularité bloquante, conformément à la Proposition IV.1 de [13].

Démonstration $d u$ Théorème I.7. Il suffit de prouver la première égalite; posons $d_{1}=\bar{c}_{1, b}-c_{0}$, et montrons $d=d_{1}$. Comme dans la démonstration $\mathrm{du}$ Théorème I.1, nous utilisons un argument de perturbation.

Pour chaque couple de bouts singuliers d'indice 1 contenus dans la même feuille, fixons dans cette feuille un chemin les reliant. Ceci fait, choisissons au voisinage des singularités $s$ d'indice $n-1$ une fonction bosse $f_{s}$ de support disjoint de ces chemins.

Approchons $\omega$ par une forme cohomologue $\omega^{\prime}=\omega-\sum \varepsilon \cdot d f_{s}$ comme dans la démonstration du Théorème I.1 (étape 3), en choisissant $\varepsilon$ de façon que toutes les composantes minimales de $\omega^{\prime}$ soient faiblement complètes, que $\mathscr{L}\left(\omega^{\prime}\right)=\mathscr{L}(\omega)$, et que $\omega^{\prime}$ n'ait pas de liaison $1 / n-1$.

Notons que $\omega$ et $\omega^{\prime}$ ont le même $K$, et en particulier le même $d_{1}$. En effet, deux bouts singuliers d'indice 1 sont sur la même feuille de $\omega^{\prime}$ si et seulement si ils sont sur la même feuille de $\omega$ : le "si" résulte du choix des supports des $f_{s}$, le "seulement si" du fait qu'on passe de $\omega$ à $\omega^{\prime}$ en coupant des feuilles près des singularités d'indice $n-1$.

Nous sommes donc ramenés à montrer $d=d_{1}$ pour $\omega^{\prime}$.

La forme $\omega^{\prime}$ n'a pas de liaison $1 / n-1$, et nous pouvons considérer le graphe $T_{\omega^{\prime}}$ comme dans l'étape 2 de la démonstration du Théorème I.1. Les sommets de $T_{\omega^{\prime}}$ se répartissent en 4 types:

1. Les composantes de $K$ dont chaque sommet est une feuille presque compacte de $\omega^{\prime}$; 
2. L'union des feuilles contenues dans l'adhérence d'une composante minimale $V_{i}$ de $\omega^{\prime}$;

3. Une union de feuilles presque compactes de $\omega^{\prime}$ ne contenant aucun bout singulier d'indice 1 ;

4. Les centres de $\omega^{\prime}$.

Considérons une composante $K_{q}$ de $K$, et sa contribution $v_{q}-1$ à $\bar{c}_{1, b}$. Si chaque sommet de $K_{q}$ est une feuille presque compacte, $K_{q}$ représente un sommet de type 1 de $T_{\omega^{\prime}}$ et $v_{q}$ est le nombre d'arêtes (orientées) de $T_{\omega^{\prime}}$ se terminant à ce sommet.

Sinon, $K_{q}$ adhère à exactement une composante minimale $V_{i}$. Comme $V_{i}$ est faiblement complète, un seul sommet de $K_{q}$ est une feuille non presque compacte. Les autres sont des feuilles presque compactes et correspondent chacun à une arête de $T_{\omega^{\prime}}$ arrivant sur le sommet de $T_{\omega^{\prime}}$ associé à $V_{i}$.

Ceci permet d'interpréter $\bar{c}_{1, b}=\sum\left(v_{q}-1\right)$ de la façon suivante. Pour chaque sommet $z$ de $T_{\omega^{\prime}}$, appelons $a_{z}$ le nombre d'arêtes (orientées) arrivant à $z$. Alors $\bar{c}_{1, b}=\sum_{(1)}\left(a_{z}-1\right)+\sum_{(2)} a_{z}$, les sommes étant prises respectivement sur les sommets de type 1 et 2 .

Soit maintenant $z$ un sommet de type 3 ou 4. Dans ce cas $a_{z}$ vaut 1 , sauf si $z$ est un centre d'indice 0: il vaut alors 0. Donc $\bar{c}_{1, b}=\sum\left(a_{z}-1\right)+$ $m+c_{0}$, où $m$ est le nombre de composantes minimales et la somme est prise sur tous les sommets de $T_{\omega^{\prime}}$. Mais $\sum\left(a_{z}-1\right)=-\chi\left(T_{\omega^{\prime}}\right)=d-m$, d'où le résultat.

I.C.ii. Persistance de singularités coniques. Nous avons montré [13, Théorème III.1] que toute forme non exacte $\omega$ est cohomologue à une forme $\omega^{\prime}$ sans singularité bloquante. Toutefois, nous allons voir que quelque chose subsiste lors du passage de $\omega$ à $\omega^{\prime}: d(\omega)$ minore le nombre de singularités d'indice 1 (resp. $n-1)$ que doit avoir une forme cohomologue à $\omega$.

Théorème I.8. Si $\omega$ et $\omega^{\prime}$ sont cohomologues, alors $c_{1}\left(\omega^{\prime}\right)$ et $c_{n-1}\left(\omega^{\prime}\right)$ sont supérieurs ou égaux à $d(\omega)$.

Remarques. -Ce théorème a été inspiré par une remarque de $\mathrm{G}$. Meigniez.

-On a en fait un résultat plus précis: $d(\omega)$ minore $c_{1}\left(\omega^{\prime}\right)-c_{0}\left(\omega^{\prime}\right)$ et $c_{n-1}\left(\omega^{\prime}\right)-c_{n}\left(\omega^{\prime}\right)$.

-La démonstration que nous allons donner est élémentaire. On peut en donner une plus conceptuelle en utilisant la théorie de l'homologie de Novikov [17], [23].

Exemple. Sur $M=\#_{p} S^{1} \times S^{n-1}$, toute forme non exacte est cohomologue à une forme $\omega$ avec $d(\omega)=p-1$. Donc toute 1-forme fermée 
de Morse possède au moins $p$ singularités d'indice 1 (resp. $n-1)$. Noter que, si $n$ est pair, il existe sur $M$ des 1 -formes différentielles intégrables non singulières [24].

Démonstration. Le cas des formes exactes (fonctions) étant bien connu, nous prenons $r(\omega) \geq 1$. Pour simplifier, nous supposons les formes sans centre. Traitons d'abord les formes de rang 1, et normalisons de façon que $P(\omega)=\mathbb{Z}$.

Sur le revêtement infini cyclique associé à $[\omega]: \pi_{1} M \rightarrow \mathbb{Z}$, les formes $\omega$ et $\omega^{\prime}$ se relèvent en des formes exactes $d f$ et $d g$, avec $f-g$ borné. Nous pouvons supposer sans perte de généralité que les points critiques de $f$ sont à des niveaux différents (cf. Remarque I.3), et que 0 est une valeur régulière de $f$ et $g$.

Soit $A$ un entier majorant $|f-g|$, et $p$ un entier $>2 A$. Définissons $X_{p}=g^{-1}([0, p])$ et $H(p)=H_{1}\left(X_{p}, g^{-1}(0) ; \mathbb{R}\right)$. Dans $X_{p}$ il y a $p \cdot c_{1}\left(\omega^{\prime}\right)$ points critiques d'indice 1 de $g$. Donc $\operatorname{dim} H(p) \leq p \cdot c_{1}\left(\omega^{\prime}\right)$.

Considérons d'autre part les $d(\omega)$ singularités bloquantes d'indice 1 de $\omega$. Appelons $x_{i}$ leurs $(p-2 A) \cdot d(\omega)$ relevés dans $f^{-1}([A, p-A]) \subset X_{p}$. Pour chaque $i$, nous appelons $L_{i}$ l'une des deux feuilles de $d f$ contenant un bout singulier de $x_{i}$, et en déduisons un homomorphisme "nombre d'intersection" $\left[L_{i}\right]: H(p) \rightarrow \mathbb{Z}$.

Nous affirmons que les $\left[L_{i}\right]$ sont linéairement indépendants dans $\operatorname{Hom}(H(p), \mathbb{R})$, ce qui ious donnera $(p-2 A) \cdot d(\omega) \leq p \cdot c_{1}\left(\omega^{\prime}\right)$ et donc $d(\omega) \leq c_{1}\left(\omega^{\prime}\right)$ puisque $p$ est arbitraire.

S'il existe une relation de dépendance $\sum a_{i}\left[L_{i}\right]=0$, considérons le $a_{i} \neq 0$ pour lequel $c_{i}=f\left(x_{i}\right)$ est minimal. L'absence de centre de $\omega$ permet de construire dans $\left.\left.f^{-1}(]-\infty, c_{i}\right]\right)$ un chemin à extrémités dans $g^{-1}(0)$, transverse à $f$ sauf en $x_{i}$, sur lequel $\left[L_{i}\right]$ vaut \pm 1 (voir Figure 3). Ceci donne $a_{i}=0$, contradiction.

Pour traiter le cas général $r(\omega) \geq 2$, il suffit de trouver arbitrairement près de $\omega$ des formes $\omega_{1}$ de rang 1 avec $d\left(\omega_{1}\right) \geq d(\omega)$. Pour cela, approchons d'abord $\omega$ par des formes $\bar{\omega}$ cohomologues, à composantes faiblement complètes, sans liaison entre singularités, avec $d(\bar{\omega})=d(\omega)$. Au moins un des deux bouts singuliers issus de chaque singularité bloquante de $\bar{\omega}$ appartient à une feuille presque compacte. Donc toute forme $\omega_{1}$ de rang 1 , vérifiant $\operatorname{Ker}[\bar{\omega}] \subset \operatorname{Ker}\left[\omega_{1}\right]$ et assez proche de $\bar{\omega}$, aura au moins autant de singularités bloquantes que $\bar{\omega}$.

I.C.iii. Quelques inégalités sur $d(\omega)$. Commençons par quelques remarques simples. Si $\omega$ ne possède pas de composante minimale faiblement complète, le groupe $\pi_{1}(M / \omega)$ est libre de rang $d+1$ et admet $P(\omega)$ 

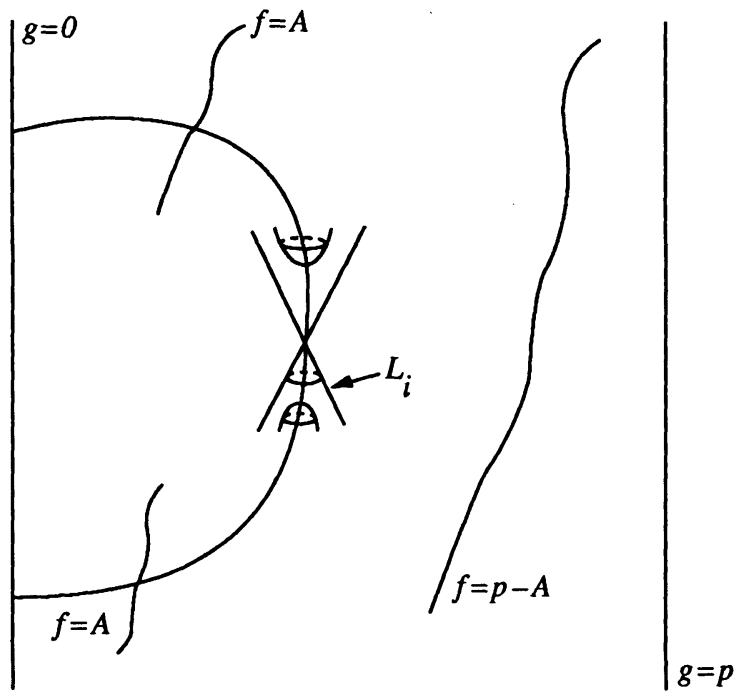

FIGURE 3

comme quotient; donc $d(\omega)+1 \geq r(\omega)$, où $r(\omega)$ est le rang de $\omega$. Notons d'autre part que $d(\omega)+1$ est toujours inférieur ou égal à $b_{1}^{\prime} M$, le rang maximal d'un quotient libre de $\pi_{1} M$ (mais qu'on construit facilement sur tout $M$ des formes $\omega$ avec $\left.d(\omega)=b_{1}^{\prime} M-1\right)$.

On en déduit par exemple, compte tenu du Théorème I.7:

Corollaire. Si $\omega$ est de rang strictement supérieur à $b_{1}^{\prime} M$, elle possède une composante minimale faiblement complète.

Corollaire. Si $\omega$ est minimale mais pas faiblement complète, elle possède au moins $2 r(\omega)-2$ singularités coniques bloquantes.

Corollaire. Génériquement dans tout $F(\Omega)$, une forme sans centre a au plus $2 b_{1}^{\prime} M-2$ singularités bloquantes.

On retrouve également un résultat de [13]: s'il n'existe pas d'épimorphisme de $\pi_{1} M$ sur $\mathbb{Z} * \mathbb{Z}$, toute forme de Morse sans centre est faiblement complète.

Considérons maintenant une forme minimale $\omega$. Nous supposons qu'elle n'est pas uniquement ergodique (donc pas faiblement complète), et nous appelons $e(\omega)$ le nombre de classes de proportionnalité de mesures transverses ergodiques de $\omega$. On sait [1, Proposition 3.4] que $e(\omega) \leq$ $b_{1}^{\prime} M-1$. En fait:

Proposition I.9. Soit $\omega$ une forme minimale non uniquement ergodique. Alors $e(\omega) \leq d(\omega)$. En particulier, $\omega$ possède au moins 4 singularités bloquantes. 
Démonstration. Elle est inspirée d'un raisonnement de [1, partie 3]. Des mesures transverses ergodiques deux à deux non proportionnelles $\mu_{1}, \cdots, \mu_{e}$ définissent des homomorphismes $\left[\mu_{i}\right]: \pi_{1} M \rightarrow \mathbb{R}$ qui se factorisent par des homomorphismes $\overline{\left[\mu_{i}\right]}: \pi_{1}(M / \omega) \rightarrow \mathbb{R}$. On montre comme dans [1] que $\overline{\left[\mu_{1}\right]}, \cdots, \overline{\left[\mu_{e}\right]}$ forment un système libre mais nor générateur dans $\operatorname{Hom}\left(\pi_{1}(M / \omega), \mathbb{R}\right)$, d'où le résultat puisque $\pi_{1}(M / \omega)$ est libre de rang $d+1$.

Corollaire. Si $\omega$ est minimale et $d(\omega) \leq 1$, alors $\omega$ est uniquement ergodique.

Remarque. Il existe $[1, \S 1.4]$ une forme $\omega$ minimale non uniquement ergodique, possédant 6 singularités coniques. Pour cette forme, $d(\omega)=3$. On ignore s'il existe des exemples avec $d(\omega)=2$.

I.D. Générateurs du pseudogroupe d'holonomie. Soit $C$ une courbe fermée transverse à une forme $\omega$. La relation d'équivalence $\sim$ donnée sur $C$ par être sur la même feuille peut être engendrée en se donnant une famille $I_{i}$ d'intervalles ouverts, une famille $R_{i}$ de rotations de $C$, et en identifiant par $R_{i}$ l'intervalle $I_{i}$ avec l'intervalle $J_{i}=R_{i}\left(J_{i}\right)$. Les restrictions $R_{i}: I_{i} \rightarrow J_{i}$ sont alors un système de générateurs du pseudogroupe d'holonomie de $\omega$ sur $C$.

Si $\omega$ est de Morse sur $M$ compacte, et si $C$ rencontre toute feuille, on montre facilement que $\sim$ peut être engendré par une famille finie de $R_{i}: I_{i} \rightarrow J_{i}$ comme ci-dessus: le pseudogroupe d'holonomie de $\omega$ sur $C$ est engendré par un nombre fini d'identifications de paires d'intervalles.

Nous nous intéressons au nombre minimum nécessaire. On peut montrer grâce à la présentation de $\pi_{1}(B \Gamma)$ donnée dans le $\S 0$. A qu'il faut au moins $b(\omega)-1$ paires, où $b(\omega)$ est le rang de l'abélianisé de $\pi_{1}(M / \omega)$ (aussi égal à son nombre minimum de générateurs).

Théorème I.10. Soit $\omega$ une forme de Morse sans centre. Supposons qu'il existe une courbe fermée transverse rencontrant toute feuille de $\omega$. Alors il en existe aussi une sur laquelle le pseudogroupe d'holonomie est engendré par $b(\omega)-1$ identifications de paires d'intervalles ouverts.

Remarques. -A priori, les intervalles que nous construirons sur la courbe transverse $C$ pourraient être égaux à $C$ ou à $C$ privé d'un point. Si $b(\omega)$ n'est pas égal à 2 , on peut en fait imposer aux $\left(I_{i}, J_{i}\right)$ d'être de vrais intervalles.

-Donnons un exemple trivial: si $\omega$ est non singulière (ou simplement faiblement complète), toute courbe transverse dont la période fait partie d'une base de $P(\omega)$ convient.

-Ce théorème permet de redémontrer le corollaire de la Proposition I.9. Soit $\omega$ minimale mais pas faiblement complète. Si $d(\omega)=1$, alors 
$\pi_{1}(M / \omega)=\mathbb{Z} * \mathbb{Z}$ et il existe une transversale sur laquelle l'holonomie est donnée par l'identification d'une paire d'intervalles. Chacun de ces intervalles doit être le cercle privé d'un point, d'où l'unique ergodicité.

Démonstration. Nous traitons successivement 3 cas, de plus en plus généraux.

1 er cas. Il n'y a pas de liaison $1 / n-1$, et toutes les feuilles régulières sont compactes. Dans ce cas, $\pi_{1}(M / \omega)$ est libre de rang $b$ et isomorphe à $\pi_{1}\left(T_{\omega}\right)$, où $T_{\omega}$ est le graphe défini dans I.A.

Puisqu'il n'y a ni centre ni liaison $1 / n-1$, l'orientation des arêtes donne sur $T_{\omega}$ une structure différentiable naturelle, pour laquelle chaque sommet est un point lisse ou un aiguillage (voir Figure 4). Les courbes transverses à $\omega$ et coupant toute feuille correspondent alors aux immersions surjectives du cercle dans $T_{\omega}$.
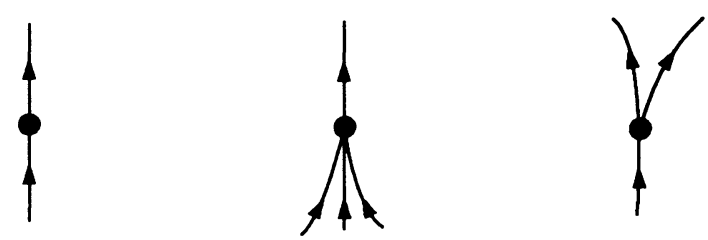

\section{FIGURE 4}

Nous sommes ainsi ramenés à un problème sur les graphes. D'une façon générale, disons qu'un graphe fini orienté $T$ est admissible si ses sommets sont comme sur la Figure 4, et s'il existe une immersion surjective du cercle dans $T$.

Soit $T$ un graphe admissible, et $\beta$ le rang de $\pi_{1} T$. Nous devons construire une immersion surjective $i: S^{1} \rightarrow T$, et $\beta-1$ homéomorphismes $\varphi_{i}$ (préservant l'orier tation) entre intervalles ouverts de $S^{1}$, de façon que $i(x)=i(y)$ si et seulement si on passe de $x$ à $y$ par applications successives des $\varphi_{i}$ ou $\varphi_{i}^{-1}$ (sauf peut-être si $i(x)=i(y)=$ un sommet de $T$ ).

Nous pouvons sans perte de généralité remplacer par un point régulier chaque sommet de $T$ à 2 arêtes. Si $\beta=1$ ou 2 , il n'y a qu'un $T$ possible et le résultat est clair (voir Figure 5). Supposons donc $\beta>2$, et raisonnons par récurrence.

Soit $T^{\prime}$ un élément maximal de l'ensemble des graphes admissibles strictement contenus dans $T$. On voit facilement que $T-T^{\prime}$ se compose d'une seule arête $a$. Puisque $\beta\left(T^{\prime}\right)=\beta(T)-1$, l'hypothèse de récurrence fournit une immersion surjective $i^{\prime}$ d'un cercle $S^{\prime}$ dans $T$. Soient $x, y$ deux points de $S^{\prime}$ s'envoyant sur les extrémités de $a$. 

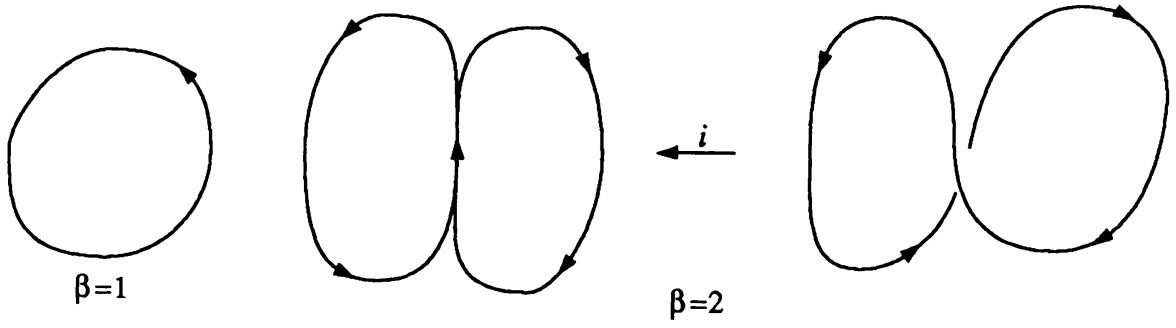

FIGURE 5

Considérons maintenant sur un cercle $S$ six points $x_{1}, y_{1}, x_{2}, y_{2}, x_{3}$, $y_{3}$ placés dans cet ordre (voir Figure 6). Soit $\tau$ une immersion de l'intervalle $\left[y_{1}, x_{1}\right] \subset S$ dans $S^{\prime}$, qui envoie chaque intervalle $\left[y_{1}, x_{2}\right],\left[y_{2}, x_{3}\right]$, $\left[y_{3}, x_{1}\right]$ sur $[y, x] \subset S^{\prime}$, et chaque intervalle $\left[x_{2}, y_{2}\right],\left[x_{3}, y_{3}\right]$ sur $[x, y]$. Définissons une immersion surjective $i: S \rightarrow T$ en envoyant $\left[x_{1}, y_{1}\right]$ sur l'arête $a$ et en posant $i=i^{\prime} \circ \tau$ sur $\left[y_{1}, x_{1}\right]$.
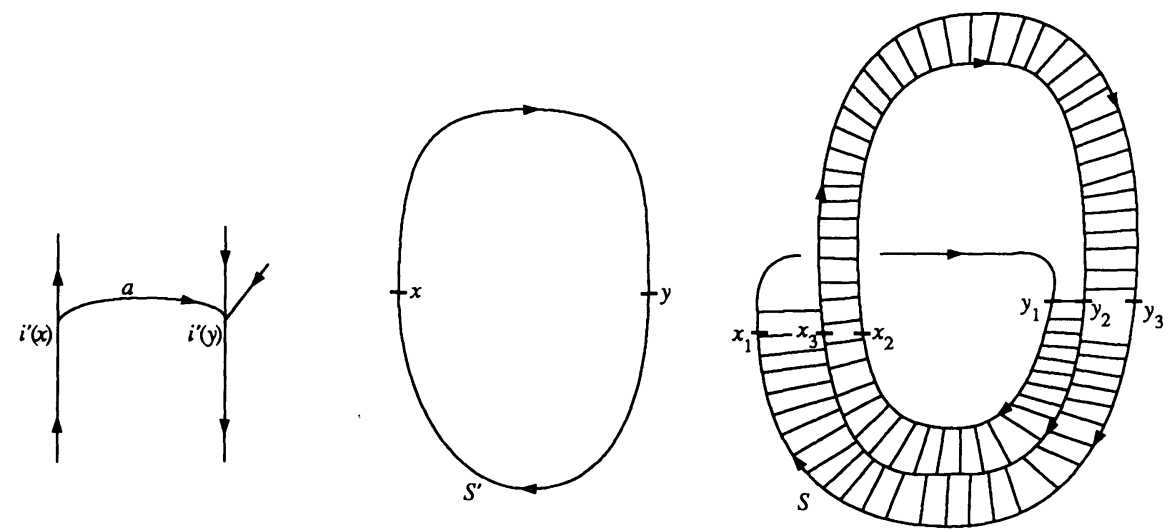

FIGURE 6

La première des $\beta-1$ identifications cherchées sur $S$ est représentée par des hachures sur la Figure 6. Elle envoie $] y_{1}, x_{3}[$ sur $] y_{2}, x_{1}[\mathrm{de}$ façon compatible avec $\tau$ (en particulier $x_{2}$ va sur $x_{3}$ et $y_{2}$ sur $y_{3}$ ).

Les $\beta-2$ autres se déduisent de celles données sur $S^{\prime}$ par l'hypothèse de récurrence. En effet, $\tau$ induit des difféomorphismes $] x_{2}, x_{3}\left[\rightarrow S^{\prime}-\{x\}\right.$ et 
]$y_{2}, y_{3}\left[\rightarrow S^{\prime}-\{y\}\right.$, qui permettent de relever à $S$ n'importe quel intervalle ouvert de $S^{\prime}$.

On vérifie que les identifications ainsi construites possèdent la propriété requise (quelle que soit la manière dont on a relevé les intervalles de $S^{\prime}$ à $S)$.

2 e cas. Il n'y a pas de liaison $1 / n-1$, et toute composante minimale est faiblement complète.

Supposons d'abord qu'il n'y a pas de feuille compacte. Sur une courbe transverse $C$ dont la période est indivisible dans $P(\omega)$, l'holonomie de $\omega$ est engendrée par $b(\omega)-1$ rotations $R_{i}$, comme demandé. Lorsque $b(\omega) \geq 2$, on peut d'ailleurs restreindre le domaine de définition des $R_{i}$ : si les intervalles ouverts $I_{i}$ recouvrent $C$, les restrictions $R_{i} \mid I_{i}$ suffisent à engendrer l'holonomie de $\omega$.

Maintenant, supposons $T_{\omega}$ homéomorphe à un cercle. Soit $C$ une courbe transverse coupant chaque feuille compacte en exactement un point. Elle traverse une fois chaque composante minimale, et nous sommes ramenés au fait suivant:

Lemme I.11. Soit $P \subset \mathbb{R}$ un sous-groupe de rang fini $r \geq 2$, et $I \subset \mathbb{R}$ un intervalle ouvert. La relation d'équivalence définie sur I par la congruence modulo $P$ peut être engendrée par $r$ identifications d'intervalles.

Démonstration. Disons que $I=] 0,1\left[\right.$. Soit $\left\{\alpha_{i}\right\} \quad(1 \leq i \leq r)$ une base de $P$, avec $0<\alpha_{i}<1$ pour tout $i$. On vérifie facilement (comparer $[1, \S 1.2])$ que les identifications $] 0,1-\alpha_{i}[\rightarrow] \alpha_{i}, 1[$ engendrent sur $I$ la congruence modulo $P$ pourvu que les $\alpha_{i}$ soient assez petits $\left(\alpha_{i}<\frac{1}{2}\right.$ suffit). q.e.d.

Si $T_{\omega}$ n'est homéomorphe ni à un point ni à un cercle, considérons un sommet $z$ correspondant à une composante faiblement complète $U$. Les feuilles $L_{j}$ de la frontière de $U$ sont presque compactes, et sont en bijection avec les arêtes touchant $z$. Elles se répartissent en deux classes, selon que l'arête arrive à $z$ ou en part.

Appelons $A$ l'ensemble des paires de feuilles $\left(L_{j}, L_{j^{\prime}}\right)$ n'appartenant pas à la même classe. Pour tout $a \in A$, soit $\sigma_{a}$ un chemin transverse joignant les deux feuilles dans $U$; nous imposons à ces chemins d'avoir des longueurs toutes distinctes (par longueur, nous entendons bien sûr la valeur absolue de l'intégrale de $\omega$ ).

Fixons de plus, pour toute paire de chemins $\left(\sigma_{a}, \sigma_{b}\right)$, un plongement $k$ du plus court dans le plus long, tel que $x$ et $k(x)$ soient sur la même feuille $\forall x$. Si $\sigma_{a}$ et $\sigma_{b}$ ont une extrémité sur une même feuille $L_{j}$, nous demandons à $k$ d'envoyer extrémité sur extrémité. 
Effectuons les choix ci-dessus successivement pour chaque composante minimale $U$, et dédoublons les sommets correspondants de $T_{\omega}$ (voir Figure 7) pour nous ramener à la situation de la Figure 4. Nous obtenons à partir de $T_{\omega}$ un graphe admissible, auquel nous appliquons le raisonnement du ler cas.
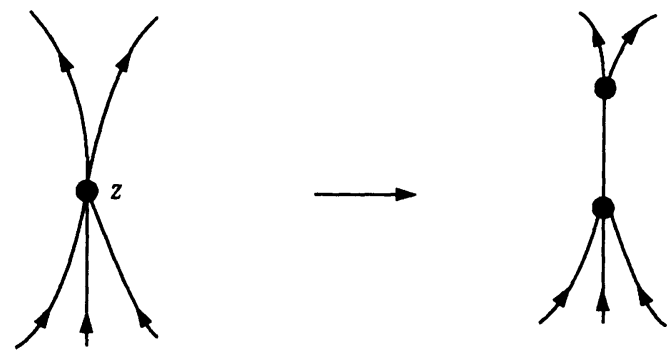

FIGURE 7

Grâce aux chemins et plongements construits ci-dessus, il vient une courbe (plongée) transverse $C$ coupant toute feuille, avec une relation d'équivalence $\sim$ possédant les propriétés suivantes: elle est engendrée par $\beta\left(T_{\omega}\right)-1$ identifications de paires d'intervalles; si $x \sim y$, alors $x$ et $y$ sont sur la même feuille; réciproquement, si $x$ et $y$ sont sur la même feuille mais pas équivalents, alors $x$ et $y$ sont équivalents à deux points d'un même $I_{U}$, où $I_{U}$ est un intervalle de longueur maximale dans $C \cap U$. On conclut alors par le Lemme I.11.

3 e cas. Cas général. Soit $C_{1}$ une courbe transverse coupant toute feuille de $\omega$. En modifiant $\omega$ près de ses singularités d'indice $n-1$, approchons-la par une forme $\omega^{\prime}=\omega-\sum \varepsilon \cdot d f_{s}$ comme dans I.C.i: en particulier, $\omega^{\prime}$ vérifie les hypothèses du 2e cas ci-dessus et $b\left(\omega^{\prime}\right)=b(\omega)$.

$\mathrm{Si} \varepsilon$ est assez petit, toute feuille de $\omega^{\prime}$ coupe $C_{1}$. Soit donc $C$ une courbe transverse à $\omega^{\prime}$, coupant toute feuille, sur laquelle le pseudogroupe d'holonomie $\Gamma_{0}$ de $\omega^{\prime}$ est donné par $b(\omega)-1$ identifications de paires d'intervalles ouverts $\left(I_{i}, J_{i}\right)$.

Nous représentons le passage de $\omega$ à $\omega^{\prime}$ sur la Figure 8 (de révolution autour d'un axe vertical); les deux formes coïncident hors d'un voisinage de la zone hachurée. En poussant $C$ hors des zones hachurées si besoin est, on peut la considérer également comme transverse à $\omega$. Identifions-la à un cercle $\mathbb{R} / \lambda \mathbb{Z}$ grâce à la mesure transverse.

Pour $0 \leq \delta \leq \varepsilon$, soit $\Gamma_{\delta}$ le pseudogroupe obtenu sur $C$ à partir de $\Gamma_{0}$ en agrandissant chaque intervalle $\left(I_{i}, J_{i}\right)$ de $\delta$ dans le sens positif (i.e. en 


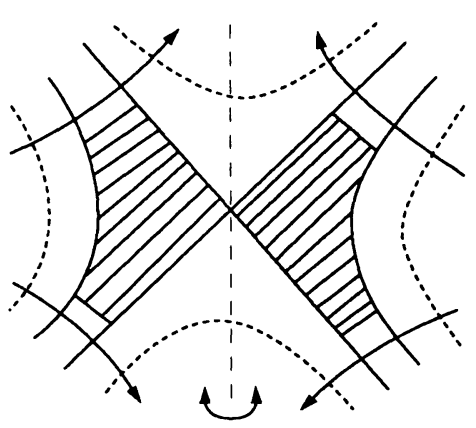

$\omega$

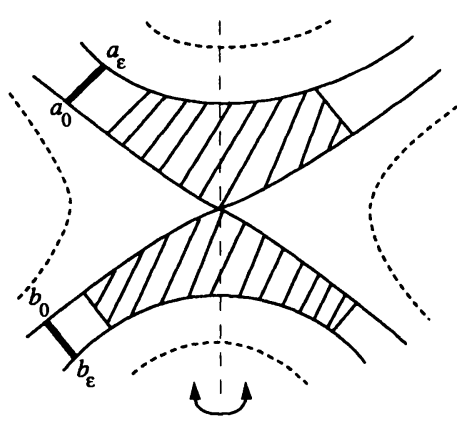

$\omega^{\prime}$

\section{FIGURE 8}

remplaçant $] p, q[$ par $] p, q+\delta[$ ). Nous allons terminer la démonstration en prouvant que $\Gamma_{\varepsilon}$ est égal au pseudogroupe d'holonomie $\Gamma$ de $\omega$.

D'abord $\Gamma_{\varepsilon} \subset \Gamma$. Soient en effet $x$ et $y$ deux points de $C$ sur la même feuille de $\omega^{\prime}$, et $\sigma$ un chemin les joignant dans cette feuille. Soit $X$ un champ de vecteurs sur $M^{*}$ tangent à $C$, vérifiant $\omega^{\prime}(X) \equiv 1$, et proportionnel à un champ hyperbolique près de Sing $\omega^{\prime}$. Lorsqu'on essaie de pousser $\sigma$ par le flot (partiellement défini) $\varphi_{t}$ de $X$, on sait (cf. [13, $\S$ II]) que le seul obstacle est constitué par les singularités d'indice $n-1$. Ceci garantit que $x+\varepsilon=\varphi_{\varepsilon}(x)$ et $y+\varepsilon=\varphi_{\varepsilon}(y)$ sont sur la même feuille de $\omega$, comme désiré.

Réciproquement, considérons au voisinage d'une singularité d'indice $n-1$ de $\omega^{\prime}$ deux intervalles transverses $\left[a_{0}, a_{\varepsilon}\right]$ et $\left[b_{0}, b_{\varepsilon}\right]$ comme sur la Figure 8. Soient $x_{t}$ et $y_{t}$ deux points de $C$ sur la même feuille de $\omega^{\prime}$ que $a_{t}$ et $b_{t}$ respectivement. Soit $A$ l'ensemble des $t \in[0, \varepsilon[$ tels que $x_{t}$ et $y_{t}$ soient dans la même orbite de $\Gamma_{\delta}$ pour tout $\left.\left.\delta \in\right] t, \varepsilon\right]$; cette définition ne dépend pas du choix de $x_{t}$ et $y_{t}$, puisque $\Gamma_{0} \subset \Gamma_{\delta}$.

On a bien sûr $0 \in A$. D'autre part, par compacité de $[0, \varepsilon]$, il existe $\alpha>0$ tel qu'on puisse choisir $x_{t}$ et $y_{t}$ continûment lorsqu'on restreint $t$ à un sous-intervalle de longueur $\leq \alpha$ dans $[0, \varepsilon]$. On en déduit que $A$ contient $t+\alpha$ dès qu'il contient $t$, d'où $A=[0, \varepsilon[$.

\section{Variétés ouvertes}

Dans toute cette partie, $\omega$ sera une 1-forme fermée non singulière sur une variété orientable sans bord $M^{n}$ (de dimension quelconque, pas 
forcément compacte). Les résultats se généralisent d'ailleurs immédiatement aux formes singulières telles que $\pi_{1} M^{*} \rightarrow \pi_{1} M$ soit un isomorphisme (par exemple si codim Sing $\omega \geq 3$ ).

II.A. Sous-groupes de type fini de $\pi_{1}(M / \omega)$.

Théorème II.1. Tout sous-groupe de type fini $H \subset \pi_{1}(M / \omega)$ est un produit libre de groupes abéliens libres, et $\rho: H \rightarrow \mathbb{R}$ est injectif sur chaque facteur de rang $\geq 2$.

Démonstration du Théorème II.1. Quitte à faire le produit par un $\mathbb{R}^{p}$, nous pouvons supposer $n \geq 4$. L'idée est de se ramener au Théorème I.1 en identifiant $H$ à $\pi_{1}\left(V / \omega_{V}\right)$, où $\omega_{V}$ est la forme (de Morse) induite par $\omega$ sur une variété compacte $V$ immergée dans $M$.

Prenons un point base $x \in M$, et fixons un sous-groupe de type fini $H \subset \pi_{1}(M, x) / \mathscr{L}(\omega)$. Considérons les immersions $i:\left(V^{n-1}, v\right) \rightarrow$ $\left(M^{n}, x\right)$, où $V$ est une variété fermée orientable de dimension $n-1$, l'image de $\pi_{1}(V, v)$ dans $\pi_{1}(M, x) / \mathscr{L}(\omega)$ est égale à $H$, et $i^{*} \omega$ est de Morse. Appelons $i_{*}$ l'épimorphisme $\pi_{1}(V, v) / \mathscr{L}\left(i^{*} \omega\right) \rightarrow H$ induit par $i$.

Puisque $n-1 \geq 3$, nous pouvons appliquer les résultats de la partie I, et en particulier définir $d\left(i^{*} \omega\right)$. Soit $d_{0}$ la valeur minimale prise par $d\left(i^{*} \omega\right)$, pour $i$ immersion comme ci-dessus.

Lemme II.2. Si $d\left(i^{*} \omega\right)=d_{0}$, alors $i_{*}: \pi_{1}(V, v) / \mathscr{L}\left(i^{*} \omega\right) \rightarrow H$ est un isomorphisme.

Modulo ce lemme, le Théorème II.1 résulte immédiatement du Théorème I.1.

Démonstration du Lemme II.2. Si le lemme est faux, il existe dans $V$ un lacet $\lambda$ basé en $v$, qui ne représente pas un élément de $\mathscr{L}\left(i^{*} \omega\right)$, alors que $i(\lambda) \in \mathscr{L}(\omega)$. Le lacet $i(\lambda)$ est donc homotope à un produit $\gamma_{1} \cdots \gamma_{k}$, où chaque $\gamma_{i}$ se compose d'un chemin $c_{i}$ partant de $x$, puis d'un lacet $\delta_{i}$ dans une feuille, puis du retour à $x$ par $c_{i}$ parcouru en sens inverse.

Supposons d'abord $k=1$. Nous pouvons alors par une homotopie régulière (fixant $v$ ) modifier $i$ dans un voisinage arbitrairement petit de $\lambda$, de façon qu'un lacet proche de $\lambda$ s'envoie sur $\delta_{1}$. Après cette opération, $\mathscr{L}\left(i^{*} \omega\right)$ a strictement augmenté, donc $d\left(i^{*} \omega\right)$ a strictement diminué (d'après le Lemme 0), contradiction.

Dans le cas général, nous allons ajouter à $V$ le bord d'un voisinage régulier de $\gamma_{k}$, de façon à diminuer $k$. De façon précise, retirons de $V$ une petite boule proche de $v$ mais disjointe de $\lambda$ et de $\operatorname{Sing}\left(i^{*} \omega\right)$, retirons également de $S^{1} \times S^{n-2}$ une petite boule, et réalisons $W=V \#\left(S^{1} \times S^{n-2}\right)$ en collant entre ces deux variétés un tube $S^{n-2} \times[0,1]$. 
Etendons $i$ en une immersion $j: W \rightarrow M$ en envoyant le tube près de $c_{k}$, et $S^{1} \times S^{n-2}$ près de $\delta_{k}$; nous supposons de plus que $j^{*} \omega$ est de Morse et qu'un cercle $\left\{S^{1} \times\right.$ point $\}$ s'envoie exactement sur $\delta_{k}$.

Nous avons ainsi $\pi_{1}(W, v) \simeq \pi_{1}(V, v) * \mathbb{Z}$, où le générateur $z \mathrm{du}$ facteur $\mathbb{Z}$ est contenu dans $\mathscr{L}\left(j^{*} \omega\right)$ et s'envoie par $j$ sur $\gamma_{k}$. Notons que l'image de $\pi_{1}(W, v)$ dans $\pi_{1}(M, x) / \mathscr{L}(\omega)$ est encore égale à $H$, et que l'application naturelle $p: \pi_{1}(V, v) / \mathscr{L}\left(i^{*} \omega\right) \rightarrow \pi_{1}(W, v) / \mathscr{L}\left(j^{*} \omega\right)$ est surjective. L'immersion $j$ est donc encore du type considéré plus haut, et $d\left(j^{*} \omega\right)=d_{0}$.

Considérons maintenant dans $W$ le lacet $\lambda \cdot z^{-1}$. Il ne peut pas être dans $\mathscr{L}\left(j^{*} \omega\right)$, car sinon $p$ n'est pas bijective et $d\left(j^{*} \omega\right)<d_{0}$ d'après le Lemme 0 . Mais $j\left(\lambda \cdot z^{-1}\right)$ est homotope à $\gamma_{1} \cdots \gamma_{k-1}:$ nous avons donc réussi à diminuer $k$.

II.B. Composantes faiblement complètes et sous-groupes abéliens. Avant d'énoncer le théorème principal de cette section, notons quelques conséquences algébriques du Théorème II.1.

Soit $H \subset \pi_{1}(M / \omega)$ un sous-groupe ne contenant pas de groupe libre $\mathbb{Z} * \mathbb{Z}$. Si $\rho$ n'est pas injectif sur $H$, alors tout sous-groupe de type fini de $H$ est cyclique. Donc $\rho(H)=\{0\}$ et $H$ est isomorphe à un sous-groupe de $\mathbb{Q}$. Nous avons montré:

Corollaire II.3. Tout sous-groupes de $\pi_{1}(M / \omega)$ ou bien contient un groupe libre non abélien, ou bien est isomorphe à un sous-groupe de $\mathbb{R}$.

Corollaire II.4. Soit $H$ un sous-groupe abélien de $\pi_{1}(M / \omega)$. L'une au moins des trois assertions suivantes est vraie:

$-\rho(H)$ est dense dans $\mathbb{R}$ et $\rho \mid H$ est injectif;

- $H$ est cyclique;

- $H$ est contenu dans $\operatorname{Ker} \rho$ et isomorphe à un sous-groupe de $\mathbb{Q}$.

Nous aurons également besoin du fait suivant:

Corollaire II.5. Les sous-groupe abéliens maximaux de $\pi_{1}(M / \omega)$ coïncident avec les centralisateurs des éléments autres que l'élément neutre. L'intersection de deux tels sous-groupes ne contient que l'élément neutre.

Démonstration. Il suffit de vérifier que le centralisateur d'un $x$ différent de l'élément neutre est abélien. Or, si $y$ et $z$ commutent avec $x$, le sous-groupe engendré par $\{x, y, z\}$ est un proligal de centre non trivial, c'est-à-dire un groupe abélien. q.e.d.

Soit maintenant $U$ une composante faiblement complète de $\omega$. L'inclusion de $U$ dans $M$ induit un homomorphisme $\pi_{1} U / \mathscr{L}(\omega \mid U) \rightarrow$ $\pi_{1}(M / \omega)$, dont le composé avec $\rho: \pi_{1}(M / \omega) \rightarrow \mathbb{R}$ est injectif d'après la Proposition I.2. 
L'image de $\pi_{1} U$ dans $\pi_{1}(M / \omega)$ est donc un sous-groupe abélien, isomorphe par $\rho$ au sous-groupe dense $P(\omega \mid U) \subset \mathbb{R}$. Nous allons montrer que ce sous-groupe est abélien maximal. En fait:

Théorème II.6. L'application qui à $U$ associe l'image $P_{U}$ de $\pi_{1} U$ dans $\pi_{1}(M / \omega)$ définit une bijection entre:

-composantes minimales faiblement complètes de $\omega$, et

-classes de conjugaison de sous-groupes abéliens maximaux d'image ( par $\rho$ ) dense dans $\mathbb{R}$.

De plus, $\rho$ établit un isomorphisme entre $P_{U}$ et $P(\omega \mid U)$.

Si $\pi_{1} M$ est de type fini, alors $\pi_{1}(M / \omega)$ est un proligal, et les classes de conjugaison ci-dessus sont en bijection avec les facteurs de rang $\geq 2$. Le Théorème II.6 généralise donc le Théorème I.1.

Corollaire. Le Théorème I.1 reste vrai pour une forme non singulière sur une variété dont le $\pi_{1}$ est de type fini.

Faisons encore quelques remarques sur le cas général, avant de montrer le Théorème II.6. En tenant compte du Théorème II.1, on obtient:

Corollaire. Si $\omega$ n'a pas de composante faiblement complète, alors $\pi_{1}(M / \omega)$ est localement libre.

Rappelons qu'un groupe est localement libre si tout sous-groupe de type fini est libre. Nous ignorons si $\pi_{1}(M / \omega)$ peut être localement libre mais pas libre.

Si $r(\omega) \leq 1$, alors $\pi_{1}(M / \omega)$ est forcément libre: dans ce cas, l'espace des feuilles est une variété non séparée de dimension 1, donc son $\pi_{1}$ est libre (ainsi que nous l'a fait remarquer A. Marin). Ceci permet de voir que $\operatorname{Ker} \rho$ est libre quel que soit le rang de $\omega$ (cf. corollaire du Théorème I.1): en effet, $\operatorname{Ker} \rho$ est le $\pi_{1}$ de l'espace des feuilles de la forme exacte induite par $\omega$ sur le revêtement de $M$ correspondant à $[\omega]: \pi_{1} M \rightarrow \mathbb{R}$.

On peut montrer d'autre part que $\pi_{1}(M / \omega)$ contient le produit libre des $P\left(\omega \mid U_{i}\right)$. Nous ne savons pas si ce sous-groupe est nécessairement un facteur libre de $\pi_{1}(M / \omega)$.

Démonstration $d u$ Théorème II.6. Nous pouvons supposer sans perte de généralité que $M$ est de dimension $n \geq 5$. Bien sûr le "de plus" est déjà démontré.

(1) Montrons d'abord que l'image de $\pi_{1} U$ dans $\pi_{1}(M / \omega)$ est un sousgroupe abélien maximal. Fixons un point base $x \in U$, une courbe transverse orientée $C \subset U$ passant par $x$, et soit $a \in \pi_{1}(M, x) / \mathscr{L}(\omega)$ commutant avec l'image de $C$.

Cette relation de commutation fournit dans $M$ une surface compacte orientable $S$ de genre 1 (tore moins des disques) telle que: 
(i) $S$ est plongée dans $M$ (ici nous utilisons $n \geq 5$ );

(ii) Chaque composante de $\delta S$ est contenue dans une feuille de $\omega$;

(iii) $S$ contient $C$;

(iv) L'image de $\pi_{1}(S, x)$ dans $\pi_{1}(M, x) / \mathscr{L}(\omega)$ est le sous-groupe engendré par $a$ et l'image de $C$.

Nous pouvons supposer que $\omega$ induit sur $S$ une forme de Morse, et que la feuille de $\omega \mid S$ passant par $x$ est régulière. Si on suit cette feuille, en partant de $x$ d'un côté de $C$, on revient nécessairement à $C$ (théorème de récurrence de Poincaré), et bien sûr par l'autre côté. Donc $a$ peut être représenté par un lacet contenu dans le saturé de $C$ pour $\omega$, c'est-à-dire dans $U$.

(2) Montrons maintenant que, si $P_{U}$ et $P_{U^{\prime}}$ sont conjugués, alors $U=$ $U^{\prime}$.

Puisque $\omega \mid U$ et $\omega \mid U^{\prime}$ ont même groupe des périodes $\rho\left(P_{U}\right)=\rho\left(P_{U^{\prime}}\right)$, nous pouvons trouver deux courbes transverses orientées $C \subset U$ et $C^{\prime} \subset$ $U^{\prime}$ ayant la même période (cf. O.B.ii). Ces courbes représentent dans $\pi_{1}(M / \omega)$ deux éléments conjugués, car $\rho$ est injective sur $P_{U}$ et $P_{U^{\prime}}$.

Il existe donc une surface compacte orientable $S_{0}$ de genre 0 , dont les bords sont d'une part $C_{1}$ et $C_{2}$, et d'autre part (peut-être) des courbes contenues dans des feuilles. Considérons la forme induite sur $S_{0}$ par $\omega$ (nous pouvons la supposer de Morse). Une feuille régulière entrant dans $S_{0}$ par un point de $C$ doit ressortir par un point de $C^{\prime}$ (récurrence de Poincaré). Donc $U=U^{\prime}$.

(3) Montrons enfin que tout sous-groupe abélien maximal $P$ avec $\rho(P)$ dense provient d'une composante faiblement complète $U$. Nous savons déjà que $\rho \mid P$ est injectif (Corollaire II.4). Fixons un point base $x \in M$, et regardons $P$ comme un sous-groupe de $\pi_{1}(M, x) / \mathscr{L}(\omega)$.

Supposons d'abord que $P$ contient un sous-groupe $P_{0}$ isomorphe à $\mathbb{Z}^{2}$. D'après le Lemme II.2, il existe une immersion $i:(V, v) \rightarrow(M, x)$, avec $i^{*} \omega$ de Morse et $\pi_{1}(V, v) / \mathscr{L}\left(i^{*} \omega\right)$ isomorphe à $P_{0}$. Le Théorème I.1 fournit dans $V$ une composante faiblement complète $U_{V}$.

Le saturé de $U_{V}$ dans $M$ est une composante minimale $U$, qui est faiblement complète d'après 0.B.ii. L'image de $\pi_{1} U$ dans $\pi_{1}(M / \omega)$ est un sous-groupe abélien maximal $P_{U}$ qui contient un conjugué de $P_{0}$. D'après le Corollaire II.5, $P_{U}$ est un conjugué de $P$.

Il reste le cas où tout sous-groupe de type fini de $P$ est cyclique. Quitte à multiplier $\omega$ par une constante, on peut supposer que $\rho$ envoie $P$ (isomorphiquement) sur un sous-groupe dense de $\mathbb{Q}$ contenant 1 . Notons que $\rho(P)$ contient alors des $1 / q$ pour $q$ arbitrairement grand (car $P$ contient $1 / q$ dès qu'il contient $p / q$ avec $p$ et $q$ premiers entre eux). 
La composante minimale $U$ cherchée sera le saturé d'une courbe transverse $C^{\prime}$ que nous allons maintenant construire. Représentons l'élément de $P$ qui s'envoie sur 1 par un lacet $C \subset M$ basé en $x$, transverse à $\omega$ sauf en un nombre fini $k$ de points, et soit $a \in P$ un élément s'envoyant sur un $1 / q$ avec $q>k$.

Puisque $a$ et $C$ commutent dans $\pi_{1}(M / \omega)$, nous pouvons trouver une surface $S$ de genre 1 vérifiant les conditions (i) à (iv) de l'étape 1 . Nous supposons encore $\omega \mid S$ de Morse, et nous notons que $P(\omega \mid S)$ est l'ensemble des multiples de $1 / q$.

Soit $L \subset S$ une feuille régulière de $\omega \mid S$, non homologue à 0 rel. $\delta S$. Comme $\int_{c} \omega=1$, le nombre d'intersection algébrique de $C$ avec $L$ dans $S$ est $\pm q$. Mais nous avons pris $q>k$ : donc $C$ contient un arc transverse à $\omega$ dont les extrémités sont sur $L$, et nous en déduisons dans $S$ une courbe fermée $C^{\prime}$ transverse à $\omega$.

Choisissons un nouveau point base $x^{\prime} \in C^{\prime}$, et regardons maintenant $P$ comme un sous-groupe de $\pi_{1}\left(M, x^{\prime}\right) / \mathscr{L}(\omega)$. Soit $r \in \mathbb{Q}$ la période de $C^{\prime}$, et soit $a^{\prime}$ un élément de $P$ s'envoyant sur un $r / q^{\prime}\left(q^{\prime} \geq 1\right)$. Construisons une surface $S^{\prime}$ de genre 1 vérifiant les conditions (i) à (iv) par rapport à $C^{\prime}, x^{\prime}$ et $a^{\prime}$, avec $\omega \mid S^{\prime}$ de Morse.

En étudiant $\omega \mid S^{\prime}$, on montre que deux points quelconques de $C^{\prime}$, dont la distance (mesurée sur $C^{\prime}$ grâce à $\omega$ ) est $r / q^{\prime}$, sont sur la même feuille de $\omega$. Puisque $q^{\prime}$ peut être arbitrairement grand, le saturé $U$ de $C^{\prime}$ pour $\omega$ est une composante minimale faiblement complète d'après $0 . \mathrm{B}$.ii, et $P_{U}=P$ d'après le Corollaire II.5.

Remarques. -Dans le cas où $P$ contient $\mathbb{Z}^{2}$, nous aurions également pu raisonner avec des surfaces de genre 1 au lieu d'utiliser l'hypersurface $V$.

-Nous verrons dans IV.B que les raisonnements ci-dessus s'étendent aux feuilletages sans holonomie, sauf le dernier (le cas où tout sous-groupe de type fini de $P$ est cyclique) qui utilise la mesure transverse de façon essentielle.

II.C. Formes faiblement complètes. Généralisant une définition de [13, p. 652], nous dirons qu'une feuille fermée $L$ de $\omega$ est triviale si $L$ sépare $M$ en deux composantes, et si $\omega$ est exacte dans l'une (au moins) de ces composantes; si $\omega$ est de rang 1, nous devons de plus considérer comme triviale une feuille $L$ telle que $M-L$ est connexe et $\omega$ est exacte dans $M-L$.

Théorème II.7. Si $\omega$ n'est pas exacte, les conditions suivantes sont équivalentes: 
(1) L'épimorphisme $\rho: \pi_{1}(M / \omega) \rightarrow P(\omega)$ est un isomorphisme.

(2) $\pi_{1}(M / \omega)$ ne contient pas de sous-groupe libre non abélien.

(3) Toute feuille n'appartenant à aucune composante minimale faiblement complète est fermée et triviale.

Si $\omega$ est exacte, (1) et (3) sont équivalents.

Remarque. Si $\omega$ est de rang $\geq 2$ et vérifie 1 , elle possède exactement une composante faiblement complète $U$, et $P(\omega \mid U)=P(\omega)$.

Une forme vérifiant la condition (1) sera dite faiblement complète. Une forme singulière $\omega$ sera faiblement complète si $\omega^{*}$ l'est. Ces définitions généralisent bien sûr celles de 0.B.ii et des parties II et VI.a de [13]. Pour une forme de Morse non exacte $\omega$ sur $M$ compacte, le faible complétude équivaut à la nullité de $d(\omega)$ et, s'il n'y a pas de centre, à l'absence de singularité bloquante.

En réunissant les Théorèmes II.1 et II.7, on obtient:

Corollaire II.8. Supposons que $\pi_{1} M$ ne contient pas $\mathbb{Z} * \mathbb{Z}$, ou que $\pi_{1} M$ est de type fini et n'a pas de quotient isomorphe $\grave{a} \mathbb{Z} * \mathbb{Z}$. Alors:

-Toute forme $\omega$ non exacte est faiblement complète;

-Aucune forme $\omega$ sur $M$ ne peut avoir de feuille exceptionnelle.

Démonstration. L'hypothèse sur $\pi_{1} M$ entraîne que $\pi_{1}(M / \omega)$ ne contient pas $\mathbb{Z} * \mathbb{Z}$. D'autre part, le Théorème II.7 implique que toute feuille est fermée ou localement dense.

Remarque. -Ce corollaire reste-t-il vrai si $\pi_{1} M$ n'a pas de quotient isomorphe à $\mathbb{Z} * \mathbb{Z}$ (mais n'est pas supposé de type fini)?

-Imanishi [10] a construit une forme non singulière $\omega$ possédant des feuilles exceptionnelles. Pour cet exemple, $\pi_{1}(M / \omega)$ est libre à une infinité de générateurs. Peut-il exister des exemples avec $\pi_{1}(M / \omega)$ de type fini? En s'inspirant du Théorème I.10, on peut également se demander si, lorsque $\pi_{1}(M / \omega)$ est de type fini, le pseudogroupe d'holonomie de $\omega$ doit être de type fini (en un sens à définir).

Démonstration $d u$ Théorème II.7. Si $\omega$ n'est pas exacte, l'équivalence entre (1) et (2) résulte des Corollaires II.3 et II.4. Supposons maintenant que $\omega$ vérifie (1) et montrons (3).

Soit $L$ une feuille non fermée (s'il en existe). Appelons $C$ une courbe transverse rencontrant $L$, et $U$ la composante faiblement complète de $\omega$. L'hypothèse sur $\rho$ entraîne l'existence dans $M$ d'une surface $S$ de genre 0 , dont les bords sont $C$, une courbe transverse contenue dans $U$, et (peut-être) des courbes contenues dans des feuilles. En regardant le feuilletage induit sur $S$, on voit que $C$, et donc $L$, est contenue dans $U$. 
Voyons maintenant que toute feuille fermée $L$ est triviale. Soit $\hat{L}$ un relevé de $L$ dans le revêtement $\hat{M}$ correspondant à l'homomorphisme $[\omega]: \pi_{1} M \rightarrow \mathbb{R}$. Dire que $L$ est triviale équivaut à dire que $\hat{L}$ sépare $\hat{M}$. Mais, si $\hat{L}$ ne sépare pas, la projection dans $M$ d'un lacet coupant $\hat{L}$ en exactement 1 point représente un élément non trivial de $\operatorname{Ker} \rho$, ce qui contredit (1).

Reste à montrer $(3) \Rightarrow(1)$. Nous laissons au lecteur le cas facile où $\omega$ est exacte; la démonstration dans ce cas revient essentiellement à voir qu'une variété (non séparée) de dimension 1 dans laquelle tout point sépare est simplement connexe. Le cas où $\omega$ est de rang 1 se ramène au précédent par passage au revêtement infini cyclique $\hat{M}$.

Soit donc $\omega$ de rang $\geq 2$ vérifiant (3). Remarquons qu'il existe au moins une composante minimale faiblement complète $U$ : sinon, toute feuille est fermée et sépare, et $\omega$ est exacte. Prenons un point base $x \in U$, fixons un élément $\alpha$ de $\operatorname{Ker}[\omega] \subset \pi_{1}(M, x)$, et représentons $\alpha$ par un lacet $\gamma:[0,1] \rightarrow M$, avec $\gamma(0)=\gamma(1)=x$, transverse à $\omega$ en dehors d'un nombre fini $k$ de points (que nous pouvons prendre hors de la frontière de $U$ ).

Notons que l'ouvert $\gamma^{-1}(U)$ a un nombre fini $(\leq k+1)$ de composantes. Si l'image de $\gamma$ est tout entière dans $U$, la Proposition I.2 donne le résultat cherché $\alpha \in \mathscr{L}$.

Sinon, soit $t_{0}$ le plus petit $t$ pour lequel $\gamma(t) \notin U$, et soit $L_{0}$ la feuille (fermée et séparante) passant par $\gamma\left(t_{0}\right)$. Appelons $t_{1}$ le plus petit $t>t_{0}$ pour lequel $\gamma(t) \in L_{0}$. Nous savons que $\gamma(t) \in U$ pour $t$ immédiatement supérieur à $t_{1}$.

D'autre part, dans la composante de $M-L_{0}$ qui ne contient pas $U$, la forme est exacte et toute feuille sépare. Le résultat étant vrai pour les formes exactes, nous pouvons modifier $\gamma$ dans un voisinage de $\left[t_{0}, t_{1}\right]$, en changeant sa classe d'homotopie par un élément de $\mathscr{L}$, de façon à diminuer le nombre de composantes de $\gamma^{-1}(U)$. Finalement nous obtenons bien $\alpha \in \mathscr{L}$.

\section{Application aux feuilletages affines}

Dans cette partie, $\mathscr{F}$ est un feuilletage non singulier de codimension 1 sur une variété $M$ orientable sans bord. Pour simplifier, nous prendrons $\mathscr{F}$ transversalement orientable.

Nous supposons $\mathscr{F}$ transversalement affine, ce qui donne une représentation d'holonomie $\pi: \pi_{1}(M) \rightarrow \operatorname{Aff}^{+}(\mathbb{R})$. Soit $H$ l'image de $\pi$, et $H_{l} \subset$ 
$\mathbb{R}^{+*}$ l'image de l'holonomie linéaire (obtenue en composant $\pi$ avec l'homomorphisme $a x+b \mapsto a)$.

Soit $p: N \rightarrow M$ le revêtement correspondant à l'holonomie linéaire; le groupe du revêtement s'identifie à $H_{l}$. Le feuilletage induit sur $N$ est défini par une 1-forme fermée $\omega$, qu'une transformation $h \in H_{l}$ multiplie par la constante $h$.

III.A. Variétés compactes. Supposons d'abord $M$ compacte. Le cas où $H$ est abélien est alors bien connu (cf. [21]). Un nombre fini le feuilles compactes découpent $M$ en ouverts $V_{i}$ dans lesquels le feuilletage peut être défini par une 1-forme fermée $\omega_{i}$; dans chaque $V_{i}$, il existe un champ de vecteurs complet $X_{i}$ avec $\omega_{i}\left(X_{i}\right) \equiv 1$, d'où une structure très simple. En particulier, il n'y a pas de feuille exceptionnelle.

Lorsque $H$ n'est pas abélien, on a:

Théorème III.1. Soit $\mathscr{F}$ un feuilletage transversalement affine sur une variété fermée orientable $M$ dont le $\pi_{1}$ ne contient pas de sous-groupe libre non abélien. On suppose que le groupe d'holonomie $H$ n'est pas abélien. Alors:

(1) Il existe un ouvert saturé connexe non vide $V$, dans lequel toute feuille est à croissance exponentielle et dense dans $V$. L'espace des feuilles $V / \mathscr{F}$ est en bijection avec $\mathbb{R} / H$.

(2) La frontière de $V$ se compose d'un nombre fini de feuilles compactes $L_{i}$. Chaque $L_{i}$ sépare $M$. Dans la composante de $M-L_{i}$ qui ne contient pas $V$, le feuilletage est à holonomie abélienne (composée uniquement d'homothéties) et les feuilles sont à croissance polynômiale.

(3) L'épimorphisme de $\pi_{1}(M / \mathscr{F})$ sur $H$ induit par $\pi$ est un isomorphisme.

Remarques. -Rappelons que $\pi_{1}(M / \mathscr{F})$ est le quotient de $\pi_{1} M$ par le sous-groupe distingué engendré par les classes d'homotopie libre de lacets contenus dans des feuilles et à holonomie triviale.

-Ce théorème montre en particulier que $\mathscr{F}$ n'a pas de feuille exceptionnelle, et que, si $\mathscr{F}$ n'a qu'une composante de Novikov (i.e. s'il existe une courbe transverse coupant toute feuille), toute feuille est dense. Il reste vrai si on suppose seulement que $\pi_{1}(M / \mathscr{F})$, et non $\pi_{1} M$, ne contient pas de sous-groupe libre non abélien; mais il peut être faux si on suppose seulement que $\pi_{1} M$ n'a pas de quotient libre non abélien. $H$.

-Si $H$ (ou même $\pi_{1} M$ ) est abélien, on n'a pas forcément $\pi_{1}(M / \mathscr{F}) \simeq$

Démonstration. Considérons la forme $\omega$ sur $N$. Puisque $H$ n'est pas abélien, le groupe des périodes $P(\omega)$ est dense dans $\mathbb{R}$. Si d'autre part $\pi_{1} M$, ou seulement $\pi_{1}(M / \mathscr{F})$, ne contient pas de sous-groupe libre 
non abélien, il en est de même de $\pi_{1}(N / \omega)$ : la forme $\omega$ vérifie donc les conditions équivalentes du Théorème II.7. Ceci montre déjà l'assertion (3) du Théorème III.1.

Soit $U$ la composante faiblement complète de $\omega$. Elle est laissée invariante par les transformations du revêtement, et $V=p(U)$ est l'ouvert cherché. En effet, l'application développante de la structure affine induit une bijection $V / \mathscr{F} \rightarrow \mathbb{R} / H$, car $U / \omega$ s'identifie à $\mathbb{R} / P(\omega)$. Si une feuille de $V$ était à croissance sous-exponentielle, il existerait pour $\mathscr{F} \mid V$ une mesure transverse invariante [18], ce qui est impossible.

Reste à montrer l'assertion (2). Soit $L \subset N$ une feuille de la frontière de $U$. On sait que $L$ est fermée, sépare $N$, et que $\omega$ est exacte dans la composante de $N-L$ qui ne contient pas $U$. Considérons un intervalle ouvert transverse coupant $L$. Il ne peut ni recouper $L$ ni couper un transformé de $L$ par $H_{l}$. Donc $p(L)$ est une feuille compacte.

S'il existait dans $M$ un lacet coupant $p(L)$ en un point, il en existerait un d'holonomie linéaire triviale, car tout élément de $H_{l}$ se représente par un lacet dans $V$; ceci empêcherait $L$ de séparer $N$. Donc $p(L)$ sépare $M$.

Soit $W$ la composante de $M-p(L)$ qui ne contient pas $V$. Un lacet dans $W$ ne peut pas avoir comme holonomie une translation non triviale. L'holonomie de $\mathscr{F} \mid W$ se compose donc d'homothéties de même centre, et les feuilles sont à croissance polynômiale d'après [2].

Enfin, les feuilles de la frontière de $V$ sont en nombre fini: comme elles séparent $M$, un intervalle ouvert transverse ne peut en rencontrer que deux.

III.B. Variétés non compactes. Le Théorème III.1 se généralise immédiatement au cas où $M$ n'est pas compacte. Les seules différences sont que les conclusions sur la croissance des feuilles n'ont plus de sens, et que la frontière de $V$ peut maintenant se composer de ce que nous appellerons une famille discrète de feuilles fermées: une union de feuilles rencontrant tout intervalle compact transverse en un ensemble fini.

Mais ce théorème de structure n'exclut pas la présence de feuilles exceptionnelles dans $M-V$ : sur une variété non compacte, un feuilletage affine à holonomie abélienne peut avoir des feuilles exceptionnelles. Cependant, nous allons montrer:

Théorème III.2. Soit $\mathscr{F}$ un feuilletage transversalement affine sur une variété $M$. Si $\pi_{1} M$ ne contient pas de sous-groupe libre non abélien, alors $\mathscr{F} n^{\prime}$ a pas de feuille exceptionnelle.

La démonstration repose sur le Corollaire II.8 et sur la remarque suivante. 
Lemme III.3. Soit $\mathscr{F}$ un feuilletage transversalement affine sur une variété $M$. Soit $X$ un ouvert saturé connexe bordé par une famille discrète de feuilles fermées, et soit $\pi_{1}(X / \mathscr{F})$ le $\pi_{1}$ de l'espace des feuilles de $\mathscr{F} \mid X$. $L$ 'application naturelle $\pi_{1}(X / \mathscr{F}) \rightarrow \pi_{1}(M / \mathscr{F})$ est injective.

Démonstration. Soit $\theta$ un lacet dans $X$ représentant l'identité dans $\pi_{1}(M / \mathscr{F})$. En supposant $M$ de dimension $\geq 5$, nous construisons une surface compacte $S$ de genre 0 , transverse à la frontière de $X$, dont le bord se compose de $\theta$ et de lacets contenus dans des feuilles et d'holonomie triviale. L'intersection de $S$ avec la frontière de $X$ se compose d'un nombre fini de courbes, qui sont toutes d'holonomie triviale. Ceci montre que $\theta$ représente l'identité dans $\pi_{1}(M / \mathscr{F})$.

Démonstration du Théorème III.2. Nous le montrons sous l'hypothèse (plus faible) que $\pi_{1}(M / \mathscr{F})$ ne contient pas de sous-groupe libre non abélien.

Ramenons-nous d'abord au cas où $H$ est abélien. Si $H$ n'est pas abélien et si $\mathscr{F}$ possède une feuille exceptionnelle $L$, il existe une feuille $L_{i}$ de la frontière de $V$ telle que $L$ appartienne à la composante $W$ de $M-L_{i}$ qui ne contient pas $V$. Dans $W$, l'holonomie est abélienne, et le $\pi_{1}$ de l'espace des feuilles ne contient pas de sous-groupe libre non abélien d'après le Lemme III.3.

Supposons donc $H$ abélien. Si $H$ est un groupe de translations, le feuilletage est défini par une 1-forme fermée, et on applique le Corollaire II.8. Sinon, $H$ est un groupe d'homothéties, disons de centre 0 . L'union des feuilles qui s'envoient sur 0 par l'application développante constitue une famille discrète de feuilles fermées. Dans chaque composante du complémentaire, le feuilletage peut être défini par une 1-forme fermée (en changeant sa structure transverse). On conclut en appliquant le Lemme III. 3 et le Corollaire II.8.

\section{Généralisation aux feuilletages sans holonomie}

IV.A. Quelques conséquences de Sacksteder. Dans cette section, nous considérons une variété fermée orientable $M^{n}(n \geq 3)$, et un feuilletage $\mathscr{F}$ de codimension 1, transversalement orienté, à singularités de Morse. Nous supposons que $\mathscr{F}$, ou plus précisément le feuilletage non singulier induit sur $M-\operatorname{Sing} \mathscr{F}$, est de classe $C^{2}$ et sans holonomie. La plupart des considérations de 0.B.i et 0.B.iii s'étendent à un tel feuilletage.

Rappelons le théorème de Sacksteder.

Théorème IV.1 [19]. Soit $\mathscr{F}$ comme ci-dessus. Si $\mathscr{F}$ n'a pas de singularité, alors $\mathscr{F}$ ne contient pas de minimal exceptionnel et est topologiquement conjugué à ( un feuilletage défini par) une 1-forme fermée. 
Le résultat peut être faux pour un feuilletage singulier, mais nous verrons (Théorème IV.7) qu'il est essentiellement vrai si $\pi_{1} M$ n'a pas de quotient libre non abélien.

Commençons par quelques définitions. Un compact invariant $K \subset M$ (i.e., l'adhérence d'une union de feuilles) est un quasiminimal e ceptionnel s'il existe une courbe transverse $C$ ne rencontrant aucune fe alle presque compacte (au sens de 0.B.iii), telle que les conditions suivantes soient vérifiées: $K$ rencontre $C$, et est minimal parmi les compacts invariants rencontrant $C$; l'intersection $K \cap C$ est un ensemble de Cantor.

On notera que $K$ peut contenir des singularités et des feuilles presque compactes, et qu'il n'existe pas de quasiminimal exceptionnel si $\mathscr{F}$ est défini par une forme fermée.

Considérons maintenant un lacet contenu dans une union finie de feuilles, sauf en un nombre fini de points qui sont des singularités coniques de même indice 1 ou $n-1$ (voir Figure 9). L'holonomie d'un tel lacet est définie d'un côté, et n'est pas forcément triviale. Si cette demi-holonomie est triviale quel que soit le lacet, nous dirons que $\mathscr{F}$ est fortement sans holonomie. C'est en particulier le cas si $\mathscr{F}$ est défini par une 1-forme fermée.

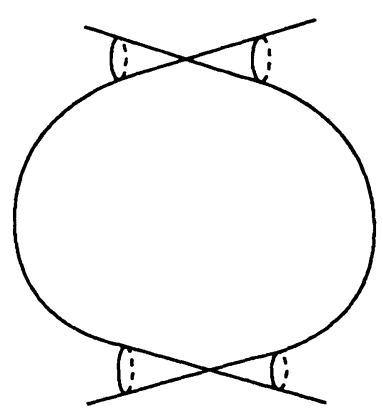

FigURE 9

L'union des feuilles qui ne sont ni compactes ni presque compactes est un ouvert $W$ formé d'un nombre fini de composantes. Soit $U$ l'une d'elles. Nous affirmons que, si $\mathscr{F}$ est fortement sans holonomie, il existe une courbe transverse $C \subset U$ rencontrant toute feuille de $\mathscr{F} \mid U$ une infinité de fois.

En effet, toute feuille de $\mathscr{F} \mid U$ est coupée par une transversale fermée. On construit une famille finie de transversales dont l'union rencontre toute feuille, et, grâce à la connexité de $U$, on arrive à une seule courbe $C$ 
coupant toute feuille. Puisque $\mathscr{F}$ est fortement sans holonomie, les feuilles de $\mathscr{F} \mid U$ ne sont pas fermées dans $U$. On en déduit qu'elles rencontrent $C$ une infinité de fois.

Ceci étant noté, les arguments de [19] donnent:

Théorème IV.2. (1) Tout quasiminimal exceptionnel $K$ contient une singularité conique bloquante s (au sens de 0.B.iii), et s'accumule simultanément sur les deux bouts singuliers de s (voir Figure 10).

(2) Le feuilletage $\mathscr{F}$ est topologiquement conjugué à une 1-forme fermée si et seulement si il est fortement sans holonomie et ne contient pas de quasiminimal exceptionnel.

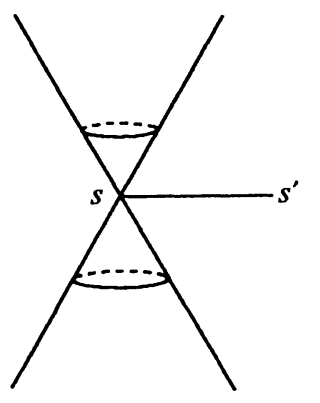

$K$ rencontre tout intervalle transverse $] s, s^{\prime}[$

FIGURE 10

Démonstration. L'assertion (1) est une conséquence directe des $\S \S 3$ et 12 de [19]. Pour l'assertion (2), soit $\mathscr{F}$ fortement sans holonomie, et sans quasiminimal exceptionnel.

Soient $U$ et $C$ comme ci-dessus. Chaque feuille de $\mathscr{F} \mid U$ doit rencontrer $C$ en un ensemble dense. Par le Théorème 4 de [19], il existe une mesure transverse invariante non triviale pour $\mathscr{F} \mid U$, qui est la restriction à $U$ d'une mesure invariante de $\mathscr{F}$ car $\mathscr{F}$ est fortement sans holonomie. Finalement, $\mathscr{F}$ possède une mesure transverse invariante donnant une mesure strictement positive à tout intervalle ouvert transverse, d'où le résultat. q.e.d.

Montrons maintenant:

Théorème IV.3. On peut, en modifiant $\mathscr{F}$ au voisinage de ses singularités coniques, l'approcher par des feuilletages topologiquement conjugués à des formes fermées (donc sans feuille exceptionnelle). 
Démonstration. Nous allons modifier $\mathscr{F}$ en coupant des feuilles au voisinage de ses singularités coniques (cf. Figure 2). Une première modification permet d'approcher $\mathscr{F}$ par un feuilletage (encore noté $\mathscr{F}$ ) fortement sans holonomie. Remarquons que les coupures à venir respecteront cette propriété.

Si $\mathscr{F}$ ne possède pas de quasiminimal exceptionnel, le Théorème IV.2 conclut. Sinon, soit $c(\mathscr{F})$ l'ensemble des singularités coniques dont les deux bouts singuliers appartiennent à une feuille presque compacte. Nous allons montrer comment approcher $\mathscr{F}$ par $\mathscr{F}^{\prime}$ avec $c(\mathscr{F})$ strictement inclus dans $c\left(\mathscr{F}^{\prime}\right)$. Cela terminera la démonstration.

Soit $K$ un quasiminimal exceptionnel, et $S$ l'ensemble des singularités bloquantes $s$ sur lesquelles $K$ s'accumule comme dans le Théorème IV.2. Puisque $\mathscr{F}$ est fortement sans holonomie, $S$ n'est pas contenu dans $c(\mathscr{F})$.

Etant donné $s \in S$, soit $] a, b[\subset] s, s^{\prime}[$ une composante du complémentaire de $K$ (voir Figure 11). Coupons des feuilles au voisinage de $s$, de façon que la feuille qui passait par $a$ devienne singulière. Nous effectuons cette opération pour chaque $s \in S$, obtenant $\mathscr{F}^{\prime}$ arbitrairement proche de $\mathscr{F}$.
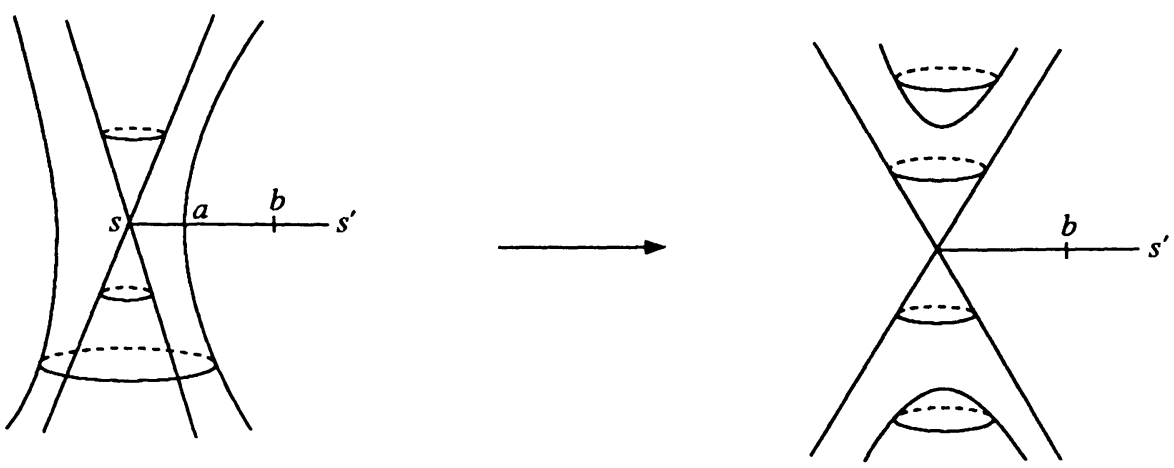

FIGURE 11

Bien sûr, $c(\mathscr{F})-S$ est contenu dans $c\left(\mathscr{F}^{\prime}\right)-S$. Il nous suffit donc de montrer $S \subset c\left(\mathscr{F}^{\prime}\right)$. Raisonnons par l'absurde. Soit $L$ une feuille non presque compacte de $\mathscr{F}^{\prime}$ contenant un bout singulier d'une $s \in S$. Soit $U$ la composante de $W$ qui contient $L$, et $C \subset U$ coupant toute feuille de $\mathscr{F}^{\prime} \mid U$ une infinité de fois (cf. ci-dessus).

L'ensemble de Cantor $K \cap C$ est l'intersection avec $C$ d'un compact $K^{\prime} \subset \bar{U}$ invariant pour $\mathscr{F}^{\prime}$. Ce $K^{\prime}$ n'est peut-être pas minimal, mais il contient un quasiminimal exceptionnel $K^{\prime \prime}$ de $\mathscr{F}^{\prime}$. Or, $\mathscr{F}^{\prime}$ a été construit pour que $K^{\prime \prime}$ contredise l'assertion (1) du Théorème IV.2. 
Corollaire IV.4. Supposons que $\mathscr{F}$ est de la forme $i^{-1}(\mathscr{G})$, où $i$ est une immersion de $M^{n}$ dans une variété de dimension $n+1$, et $\mathscr{G}$ est un feuilletage non singulier, de classe $C^{2}$, sans holonomie. Alors $\mathscr{F}$ est topologiquement conjugué à une 1-forme fermée.

Démonstration. Les singularités de $\mathscr{F}$ sont les points où $i$ n'est pas transverse à $\mathscr{G}$. Une modification de $i$ au voisinage d'une singularité conique $s$, déplaçant transversalement à $\mathscr{G}$ le point de contact, revient à couper ou recoller des feuilles au voisinage de $s$. Le feuilletage $\mathscr{F}$ s'obtient donc à partir d'un feuilletage sans holonomie $\mathscr{F}^{\prime \prime}$ en coupant des feuilles près de chaque singularité conique.

D'après le Théorème IV.3, il existe près de $\mathscr{F}^{\prime \prime}$ un feuilletage $\mathscr{F}^{\prime}$ conjugué à une forme fermée. Si $\mathscr{F}^{\prime}$ est assez proche de $\mathscr{F}^{\prime \prime}$, le feuilletage $\mathscr{F}$ sera obtenu à partir de $\mathscr{F}^{\prime}$ par coupures de feuilles, et sera donc conjugué à une forme fermée.

IV.B. Relations avec le $\pi_{1}$. Le Corollaire IV.4 va nous permettre d'étudier les feuilletages sans holonomie des variétés ouvertes. Bien entendu, les résultats obtenus peuvent à leur tour être appliqués aux feuilletages singuliers considérés ci-dessus. C'est ainsi que le Théorème IV.7 impliquera le Théorème 11 énoncé en début d'article.

Dans cette section, $M$ sera donc une variété orientable quelconque, et $\mathscr{F}$ un feuilletage non singulier de codimension 1 , transversalement orientable, de classe $C^{2}$, sans holonomie.

Notons d'abord le fait suivant, conjecturé par Lamoureux [12]:

Théorème IV.5. Un feuilletage $\mathscr{F}$ comme ci-dessus n'admet pas de transversale fermée $C$ homologue à 0 .

Démonstration. Raisonnons par l'absurde. Nous pouvons supposer sans perte de généralité que $M$ est de dimension $n \geq 5$. Il existe alors une variété fermée $V^{n-1}$, une courbe $C^{\prime} \subset V$ homologue à 0 dans $V$, et une immersion $i: V \rightarrow M$ induisant un difféomorphisme de $C^{\prime}$ sur $C$. En supposant $i^{-1}(\mathscr{F})$ de Morse, le Corollaire IV.4 donne la contradiction cherchée, puisqu'une forme fermée n'a pas de transversale homologue à 0 . q.e.d.

Voici une autre conséquence du Corollaire IV.4. En raisonnant exactement comme dans II.A, on obtient:

Théorème IV.6. Tout sous-groupe de type fini $H \subset \pi_{1}(M / \mathscr{F})$ est un produit libre de groupes abéliens libres. Si aucune feuille de $\mathscr{F}$ n'est localement dense, alors $H$ est libre.

Montrons maintenant le résultat principal de cette section:

Théorème IV.7. Si $\pi_{1}(M / \mathscr{F})$ ne contient pas de sous-groupe libre non abélien, et n'est pas isomorphe à un sous-groupe dense de $\mathbb{Q}$, alors $\mathscr{F}$ n'a pas de feuille exceptionnelle. 
Remarques. -Ce théorème s'applique en particulier si $\pi_{1} M$ est de type fini et n'a pas de quotient libre non abélien.

-Comme nous l'avons remarqué au début de l'article, les deux conditions sur $\pi_{1}(M / \mathscr{F})$ sont nécessaires.

Démonstration. Nous distinguons deux cas.

1 er cas. $\pi_{1}(M / \mathscr{F})$ est trivial ou infini cyclique. Dans ce cas, nous allons voir que toute feuille est propre (comparer [11]).

Notons d'abord que dans un feuilletage sans holonomie (ou plus généralement à holonomie analytique), une transversale fermée $C$ ne peut pas être triviale dans $\pi_{1}(M / \mathscr{F})$ : c'est le raisonnement de Haefliger [6, pp. 390-392], appliqué sur une surface de genre 0 bordée par $C$ et des lacets d'holonomie triviale (contenus dans des feuilles de $\mathscr{F}$ ).

Si $\pi_{1}(M / \mathscr{F})$ est trivial, toute feuille est donc fermée. Si $\pi_{1}(M / \mathscr{F}) \simeq$ $\mathbb{Z}$, considérons le revêtement infini cyclique $p$ correspondant. Soit $L$ une feuille de $\mathscr{F}$, et $C$ une transversale fermée. Une composante de $p^{-1}(L)$ et une composante de $p^{-1}(C)$ se coupent au plus une fois. Ceci montre que $L$ coupe $C$ au plus $n$ fois, où $n>0$ est la valeur de $C$ dans $\pi_{1}(M / \mathscr{F}) \simeq \mathbb{Z}$. La feuille $L$ est donc propre.

$2 e$ cas. D'après le Théorème IV.6, c'est celui où $\pi_{1}(M / \mathscr{F})$ est abélien et contient $\mathbb{Z}^{2}$. Nous affirmons alors que toute feuille est fermée ou localement dense.

Nous allons généraliser des raisonnements de II.B et II.C, grâce à la remarque suivante. Soit $S \subset M$ une surface compacte de genre 0 , avec $\mathscr{F} \mid S$ de Morse et $\delta S$ composé de courbes transverses à $\mathscr{F}$ et de courbes contenues dans des feuilles; alors toute feuille de $\mathscr{F} \mid S$ partant de $\delta S$ doit retourner à $\delta S$ ou aboutir à une selle.

Puisque $\pi_{1}(M / \mathscr{F})$ contient $\mathbb{Z}^{2}$, il existe une immersion $i: V^{n-1} \rightarrow$ $M^{n}$ avec $V$ fermée, $\mathscr{H}=i^{-1}(\mathscr{F})$ de Morse, et $\pi_{1}(V / \mathscr{H}) \simeq \mathbb{Z}^{2}$. Le feuilletage $\mathscr{H}$ est topologiquement conjugué à une forme fermée possédant une composante minimale faiblement complète.

Prenons dans (l'image de) cette composante une courbe $C$ transverse à $\mathscr{H}$. Nous l'identifions à un cercle (par un homéomorphisme), de façon que le pseudogroupe d'holonomie de $\mathscr{H}$ sur $C$ contienne une rotation sans point périodique.

Soit $U$ le saturé de $C$ pour $\mathscr{F}$. On montre comme dans II.B que $\pi_{1} U$ engendre $\pi_{1}(M / \mathscr{F})$. Puisque $\mathscr{F}$ est sans holonomie, on voit facilement que le pseudogroupe d'holonomie de $\mathscr{F}$ sur $C$ préserve la mesure de Lebesgue, et donc (Lemme III.5 de [13]) qu'il est engendré par un groupe de rotations. 
En d'autres termes, $U$ se comporte comme une composante minimale faiblement complète. En particulier, tout élément non trivial de $\pi_{1}(M / \mathscr{F})$ est représentable par une transversale fermée contenue dans $U$. On montre alors comme dans II.C que toute feuille non fermée est dans $U$.

\section{Application aux variétés non séparées de dimension 1}

Si $\omega$ est une forme non singulière sur une variété $M$, nous avons remarqué dans 0 .A que $\pi_{1}(M / \omega)$ agit librement sur une 1-variété simplement connexe $V_{\mathscr{L}}$. Réciproquement, nous allons associer à des actions de certains groupes $G$ sur des 1-variétés un feuilletage sans holonomie $\mathscr{F}$ tel que $G \simeq \pi_{1}(M / \mathscr{F})$. Ceci nous permettra d'appliquer les résultats de la partie IV et de montrer:

Théorème V.1. Soit $V$ une variété connexe et simplement connexe de dimension 1. Soit $G$ un groupe de type fini agissant sur $V$ par $C^{2}$ difféomorphismes, librement et en préservant l'orientation. Alors $G$ est un produit libre de groupes abéliens libres. Si aucune orbite n'est localement dense dans $V$, alors $G$ est libre.

Remarque. Si $G$ n'est pas de type fini, on peut appliquer ce théorème à tous ses sous-groupes de type fini. On montre d'autre part que $G$ ou bien contient $\mathbb{Z} * \mathbb{Z}$ ou bien est isomorphe à un sous-groupe de $\mathbb{R}$.

Démonstration. Fixons sur $V$ une orientation et une métrique riemannienne. Soit $f: V \rightarrow \mathbb{R}$ un développement, c'est-à-dire une immersion isométrique préservant l'orientation; une telle $f$ est bien déterminée modulo composition avec une translation de $\mathbb{R}$.

Soit $U_{i}$ une famille dénombrable d'intervalles ouverts recouvrant $V$, soit $U$ l'union disjointe des $U_{i}$, et soit $\pi: U \rightarrow V$ l'application naturelle. Considérons la variété (non connexe) $U \times \mathbb{R}^{n} \quad(n \geq 3)$, munie de la forme exacte $d(f \circ \pi \circ p)$, où $p$ est la projection de $U \times \mathbb{R}^{n}$ sur $U$.

Soit $(i, j)$ un couple tel que $U_{i}$ et $U_{j}$ se rencontrent dans $V$. Leur intersection est alors un intervalle (car $V$ est simplement connexe), qui se relève en deux intervalles ouverts $I_{i j} \subset U_{i}$ et $J_{i j} \subset U_{j}$. Fixons dans $\mathbb{R}^{n}$ une petite boule fermée $B_{i j}$, et ôtons de $U \times \mathbb{R}^{n}$ les ouverts $I_{i j} \times$ int $B_{i j}$ et $J_{i j} \times$ int $B_{i j}$, ainsi que les compacts (peut-être vides) $\delta I_{i j} \times B_{i j}$ et $\delta J_{i j} \times B_{i j}$. La variété obtenue a deux bords $I_{i j} \times \delta B_{i j}$ et $J_{i j} \times \delta B_{i j}$, que nous recollons par l'identification naturelle.

Effectuons cette chirurgie pour chaque couple $(i, j)$, en choisissant des $B_{i j}$ éloignées les unes des autres dans $\mathbb{R}^{n}$. Nous obtenons une variété $N$ munie d'une forme exacte $\theta$. 
Soit $x \in \mathbb{R}^{n}$ n'appartenant à aucune $B_{i j}$. Alors $U \times\{x\}$ définit un plongement de $U$ dans $N$ transverse à $\theta$. La construction a été faite pour que $U$ rencontre toute feuille de $\theta$, et que deux points de $U$ appartiennent à la même feuille si et seulement si ils ont la même image dans $V$. En particulier, $\theta$ est faiblement complète: puisque dans $V$ tout point sépare, toute feuille de $\theta$ sépare $N$.

Oublions maintenant la métrique sur $V$, et faisons intervenir l'action de $G$. Pour chaque $g \in G$, choisissons une famille dénombrable d'applications $\gamma(g, k): I(g, k) \rightarrow J(g, k)$ entre intervalles ouverts de $U$ de façon que, étant donné $a$ et $b$ dans $U$, il existe un $k$ avec $b=\gamma(g, k)(a)$ si et seulement si $\pi(b)=g \cdot \pi(a)$.

Pour chaque $(g, k)$, la donnée de $\gamma(g, k)$ nous permet d'effectuer une chirurgie sur $N$ comme ci-dessus. Puisque $G$ préserve l'orientation sur $V$, les $\gamma(g, k)$ préservent sur $U$ l'orientation induite par $\pi$; la variété $M$ obtenue après ces chirurgies est donc munie d'un feuilletage transversalement orienté $\mathscr{F}$. L'action de $G$ étant libre et $C^{2}, \mathscr{F}$ est sans holonomie et $C^{2}$.

Il y a encore un plongement de $U$ dans $M$ transverse à $\mathscr{F}$ et rencontrant toute feuille, mais maintenant deux points de $U$ sont sur la même feuille si et seulement si leurs images par $\pi$ sont dans la même orbite de $G$.

Nous affirmons que $\pi_{1}(M / \mathscr{F}) \simeq G$. Remarquons d'abord que $\pi_{1} M$ peut ètre vu comme le produit libre de $\pi_{1} N$ avec le groupe libre engendré par les lettres $\gamma(g, k)$. On définit alors un épimorphisme $\lambda$ de $\pi_{1} M$ dans $G$ en envoyant $\pi_{1} N$ sur 1 et $\gamma(g, k)$ sur $g$. Compte tenu du fait que $\theta$ est faiblement complète, on voit facilement que le noyau de $\lambda$ est contenu dans $\mathscr{L}(\mathscr{F})$. D'autre part un élément de $\mathscr{L}(\mathscr{F})$ s'envoie dans $G$ sur un élément dont l'action a un point fixe, c'est-à-dire sur 1 puisque l'action est libre.

Le groupe $G$ est donc un proligal d'après le Théorème IV.6. Si aucune orbite n'est localement dense, aucune feuille de $\mathscr{F}$ n'est localement dense, et $G$ est libre.

\section{Références}

[1] P. Arnoux \& G. Levitt, Sur l'unique ergodicité des 1-formes fermées singulières, Invent. Math. 84 (1986) 141-156.

[2] R. A. Blumenthal, Transversely homogeneous foliations, Ann. Inst. Fourier (Grenoble) 29 (1979) 143-158.

[3] T. Cherry, Analytic quasi-periodic curves of discontinuous type on a torus, Proc. London Math. Soc. 44 (1938) 175-215. 
[4] D. I. Fouxe-Rabinovitch, On the determinators of an operator of the free group, Mat. Sb. 7 (1940) 197-206.

[5] K. W. Gruenberg, Residual properties of infinite soluble groups, Proc. London Math. Soc. 7 (1957) 29-62.

[6] A. Haefliger, Variétés feuilletées, Ann. Scuola Norm. Sup. Pisa Cl. Sci. (4) 16 (1962) 367-397.

[7] __ Groupoïdes d'holonomie de classifiants, in Structures Transverses des Feuilletages, Astérisque 116 (1984) 70-97.

[8] J. Hempel, 3-manifolds, Ann. of Math. Studies, No. 86, Princeton University Press, Princeton, NJ, 1976.

[9] H. Imanishi, On codimension one foliations defined by closed one forms with singularities, J. Math. Kyoto Univ. 19 (1979) 285-291.

[10] _ Structure of codimension 1 foliations without holonomy on manifolds with abelian fundamental group, J. Math. Kyoto Univ. 19 (1979) 481-495.

[11] C. Lamoureux, The structure of foliations without holonomy on non-compact manifolds with fundamental group $\mathbb{Z}$, Topology 13 (1974) 219-224.

[12] __ Feuilletages des variétés compactes et non compactes, Ann. Inst. Fourier (Grenoble) 26 (1976) 221-271.

[13] G. Levitt, 1-formes fermées singulières et groupe fondamental, Invent. Math. 88 (1987) 635-667.

[14] R. C. Lyndon, Length functions in groups, Math. Scand. 12 (1963) 209-234.

[15] W. Magnus, A. Karrass \& D. Solitar, Combinatorial group theory, Pure and Applied Math., Vol. 13, Interscience, New York, 1966, 245.

[16] J. Morgan \& P. Shalen, Valuations, trees, and degeneration of hyperbolic structures. I, Ann. of Math. (2) 120 (1984) 401-476.

[17] S. P. Novikov, Multivalued functions and functionals; an analogue of the Morse theory, Soviet Math. Dokl. 24 (1981) 222-226.

[18] J. F. Plante, Foliations with measure preserving holonomy, Ann. of Math. (2) 102 (1975) 327-361.

[19] R. Sacksteder, Foliations and pseudogroups, Amer. J. Math. 87 (1965) 79-102.

[20] E. Salem, Riemannian foliations and pseudogroups of isometries, appendix D in P. Molino, Riemannian Foliations, Progress in Math., Vol. 73, Birkhäuser, Boston, 1988.

[21] B. Seke, Sur les structures transversalement affines des feuilletages de codimension un, Ann. Inst. Fourier (Grenoble) 30 (1980) 1-29.

[22] P. Shalen, Dendrology of groups, Essays in Group Theory (Gersten, ed.), Math. Sci. Res. Inst. Publ., Vol. 8, Springer, Berlin and New York, 1987, 265-319.

[23] J. C. Sikorav, Homologie de Novikov associée à une classe de cohomologie réelle de degré un, Thèse d'êtat, Orsay, 1987.

[24] W. P. Thurston, Existence of codimension-one foliations, Ann. of Math. (2) 104 (1976) 249-268.

[25] D. Tischler, On fibering certain foliated manifolds over $S^{1}$, Topology 9 (1970) 153-154.

\author{
Université PARIS SUd \\ Université Paul Sabatier, Toulouse
}


